\title{
Monopole versus spherical harmonic superconductors: Topological repulsion, coexistence, and stability
}

\author{
Enrique Muñoz $\odot,{ }^{1,2}$ Rodrigo Soto-Garrido $\odot,{ }^{1}$ and Vladimir Juričić $\oplus^{3}$ \\ ${ }^{1}$ Facultad de Física, Pontificia Universidad Católica de Chile, Vicuña Mackenna 4860, Santiago, Chile \\ ${ }^{2}$ Center for Nanotechnology and Advanced Materials CIEN-UC, Avenida Vicuña Mackenna 4860, Santiago, Chile \\ ${ }^{3}$ Nordita, KTH Royal Institute of Technology and Stockholm University, Roslagstullsbacken 23, 10691 Stockholm, Sweden
}

(Received 28 July 2020; accepted 30 October 2020; published 13 November 2020)

\begin{abstract}
The monopole harmonic superconductor (SC), proposed in doped Weyl semimetals as a pairing between the Fermi surfaces enclosing the Weyl points, is rather unusual, as it features the monopole charge inherited from the parent metallic phase. However, this state can compete with more conventional spherical harmonic pairings, such as an $s$ wave. We here demonstrate, within the framework of the weak-coupling mean-field BCS theory, that the monopole and a conventional spherical harmonic SC quite generically coexist, while the repulsion can take place when the absolute value of the monopole charge matches the angular momentum quantum number of the spherical harmonic. As we show, this feature is a direct consequence of the topological nature of the monopole SC, and we dub it topological repulsion. We illustrate the above principle with the example of the conventional $s$ - and $\left(p_{x} \pm i p_{y}\right)$-wave pairings competing with the monopole $\mathrm{SC} Y_{-1,1,0}(\theta, \phi)$, which coexist in a finite region of the parameter space and repel, respectively. Furthermore, the $s$-wave pairing is more stable both when the chemical potentials at the nodes are unequal and in the presence of pointlike charged impurities. Since the phase transition is discontinuous, close to the phase boundary, we predict that the Majorana surface modes at the interfaces between domains featuring the monopole and the trivial phases, such as an $s$-wave, will be the experimental signature of the monopole SC.
\end{abstract}

DOI: 10.1103/PhysRevB.102.195121

\section{INTRODUCTION}

Topological semimetals feature the nodal points in the Brillouin zone where the conduction and valence bands touch, yielding a rather rich landscape of emergent lowenergy quasiparticles [1-6]. In particular, the exotic electronic properties in Weyl semimetals (WSMs), such as Fermi arc surface states and anomalous magnetotransport, arise from the two topological nodal points in the Brillouin zone featuring pseudorelativistic Weyl fermions [7-11], which were experimentally observed in mostly binary compounds, such as TaAs and $\mathrm{NbP}$ [12-15]. These Weyl points are the source and the sink of the Abelian Berry curvature, yielding the monopole charge $\mathcal{C}= \pm 1$, the topological invariant characterizing these semimetals. Weyl metals can also represent a platform for the realization of yet different states of matter. For instance, they can host an interaction-driven fully gapped axionic insulator [16-19], which was recently experimentally observed [20]. On the superconducting side, WSMs can accommodate a plethora of pairing states [21-27]. The monopole superconductor (SC), recently proposed as a pairing state between the two Fermi surfaces (FSs) enclosing the Weyl points in a doped

Published by the American Physical Society under the terms of the Creative Commons Attribution 4.0 International license. Further distribution of this work must maintain attribution to the author(s) and the published article's title, journal citation, and DOI. Funded by Bibsam.
WSM [28], is an exciting possibility because it hosts vortices inherited from the underlying WSM state, but its physical consequences have been only touched upon so far [29].

An urgent issue in this respect is the competition of the monopole pairing, characterized by the monopole harmonic functions $Y_{q, j, m}(\theta, \phi)$, with more conventional spherical harmonic states $Y_{j, m}(\theta, \phi)$, as well as its stability in the presence of impurities. We here demonstrate, within the framework of the mean-field BCS theory, that the monopole SC and a conventional spherical harmonic phase quite generically can coexist with one another, while the repulsion takes place when the $\theta$-dependent form factors of the monopole harmonic $Y_{|q|, j,|m|}(\theta, \phi)$ and the spherical harmonic $Y_{q=0, j, m}(\theta, \phi) \equiv$ $Y_{j, m}$ are proportional. As we show, this manifestly gaugeindependent feature is a direct consequence of the topological nature of the monopole superconductor, and we dub it topological repulsion. In particular, this mechanism implies that a monopole harmonic with the charge $q>0, Y_{-q, q, 0}(\theta, \phi)$ and the spherical harmonic $Y_{q, q}(\theta, \phi)$ always repel, with the coexistence possible only at the phase boundary. We illustrate the above principle by showing that the conventional $s$ wave and a monopole superconductor $Y_{-1,1,0}$ can coexist in a finite region of the parameter space, as shown in Fig 1(a). On the other hand, this monopole pairing repels the $p_{x} \pm i p_{y}$ superconducting states, as displayed in Fig. 1(b). Furthermore, the $s$-wave pairing is more stable both when the chemical potentials at the nodes are unequal (Fig. 2) and in the presence of pointlike charged impurities (see Fig. 3). Since the phase transition is discontinuous, close to the phase boundary, we predict that the Majorana surface modes at the interfaces between domains 

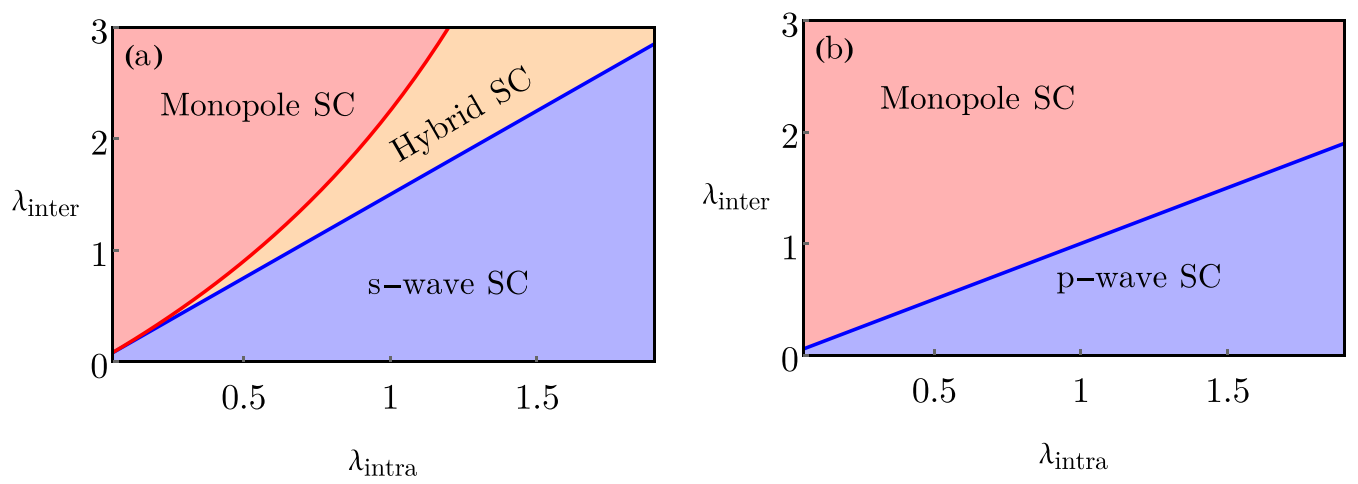

FIG. 1. Zero-temperature phase diagram of the Weyl superconductor (SC) with the inter- and intra-Fermi surface pairings tuned by the couplings $\lambda_{\text {inter }}$ and $\lambda_{\text {intra }}$, respectively. (a) Monopole versus the $s$-wave pairing. The two phases coexist in a finite region of the phase diagram (hybrid SC). The phase boundaries between mixed and pure phases are given by Eq. (27) (blue solid line) and Eq. (28) (red solid line). (b) Monopole versus the $p_{ \pm}$-wave SC. Topological repulsion is operative; thus, the phases may coexist only at the phase boundary where the two couplings are equal; see the discussion after Eq. (17) and Sec. III B. The phase transitions between different phases are discontinuous. The effective couplings $\lambda_{\text {inter }}$ and $\lambda_{\text {intra }}$ are defined in Eq. (13).

featuring the monopole and the trivial phases, such as an $s$ wave, will be the experimental signature of the monopole superconductor in the system.

The rest of the paper is organized as follows. In Sec. II, we present the details of the continuum model for the Weyl semimetal. Section III is devoted to the mean-field analysis of the competition and coexistence between the monopole and spherical harmonic pairings corroborated by a gauge invariance argument for the topological repulsion. In Sec. IV, we analyze the effects of the impurity scattering on the competing $s$-wave and monopole SCs. Finally, in Sec. V, we discuss our results, and we present necessary technical details in the Appendixes.

\section{MODEL}

We start by considering the model describing the meanfield Cooper pairing between the Weyl quasiparticles living at the FSs enclosing the two nodal points with opposite monopole charges $\mathcal{C}_{ \pm}= \pm 1$ :

$$
\hat{H}=\hat{H}_{\text {Weyl }}+\hat{H}_{\Delta} \text {. }
$$

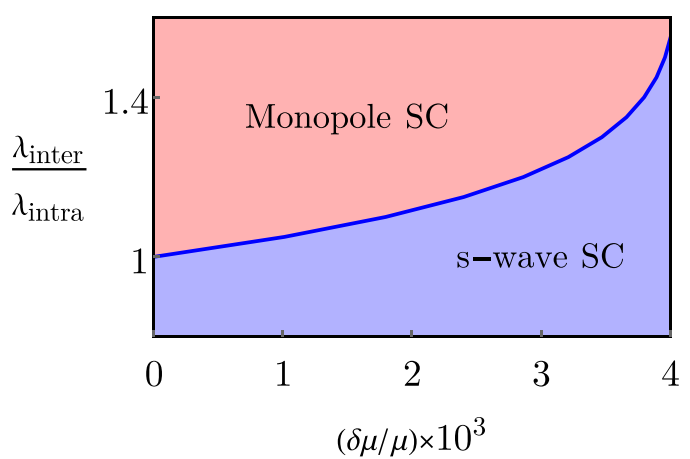

FIG. 2. Phase diagram of a Weyl superconductor with the difference between the chemical potentials $\delta \mu$ at the nodes (for $\lambda_{\text {intra }}=$ 0.2 ). The $s$ wave is more favorable in this case. The phase diagram is obtained from the behavior of the phase boundary close to the critical temperature from Eqs. (C23) and (C24).
The continuum Hamiltonian corresponding to the timereversal symmetry-breaking WSM with the two nodal points is $\hat{H}_{\text {Weyl }}=\sum_{\zeta= \pm, \mathbf{q}} \hat{c}_{\zeta \mathbf{K}_{0}+\mathbf{q}}^{\dagger} \hat{h}_{\zeta}(\mathbf{q}) \hat{c}_{\zeta \mathbf{K}_{0}+\mathbf{q}}$, where

$$
\hat{h}_{\zeta}(\mathbf{q})=v_{F}\left(\sigma_{x} q_{x}+\sigma_{y} q_{y}+\zeta \sigma_{z} q_{z}\right)-\mu,
$$

and the chemical potential $\mu>0$. This Hamiltonian is obtained after expanding the corresponding lattice model about the two Weyl nodes along the $k_{z}$ direction located at $\zeta \mathbf{K}_{0}=$ $\left(0,0, \zeta K_{0}\right)$ (see Appendix A). We here consider only isotropic nodes with Fermi velocity $v_{F}=1$ and fix the position of the nodes at $K_{0}=\pi / 2 a$, with the lattice constant $a=1$, and also $\hbar=k_{B}=1$ hereafter.

We here adopt a phenomenological model in which we assume both inter-FS and intra-FS pairing interactions without delving into microscopic details yielding such interactions.

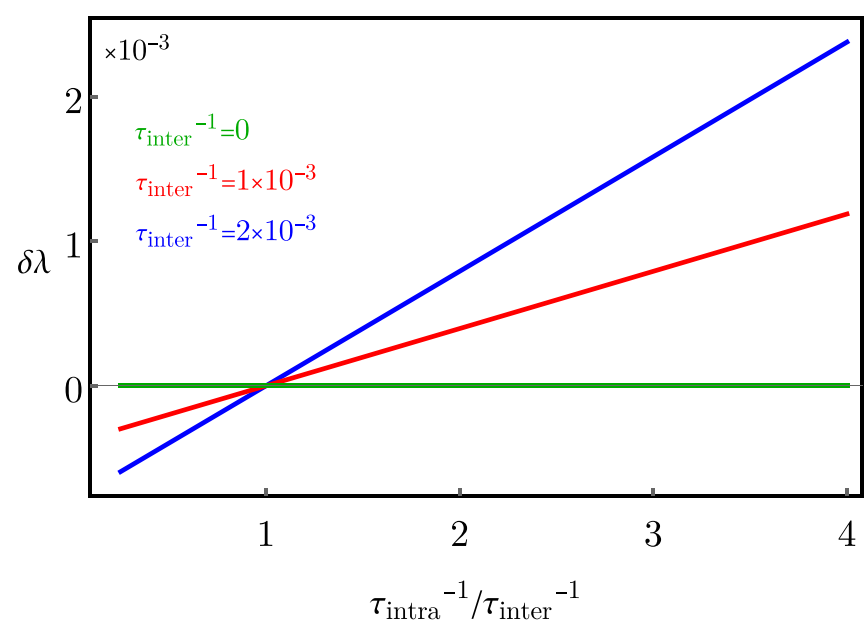

FIG. 3. The ratio of the inter- and intra-Fermi surface couplings at the phase boundary (two critical temperatures are equal) between the $s$ wave and the monopole superconductor in the presence of inter- and intra-Fermi surface scattering by the point impurities. The respective scattering times are $\tau_{\text {inter }}$ and $\tau_{\text {intra }}$ (in units of $1 / \mu$ ). The change in the phase boundary shows that the $s$-wave pairing is more stable. Here, $\delta \lambda=\lambda_{\text {inter }} / \lambda_{\text {intra }}-1$, and $\lambda_{\text {inter }}=0.13$. 
This allows us to address the universal aspects of the competition and coexistence between the spherical and monopole harmonics in a Weyl superconductor, which is the main purpose of this paper. The Hamiltonian for an inter-FS $s$-wave spin-singlet pairing is

$$
\hat{H}_{\Delta}=\sum_{\mathbf{q}} \hat{c}_{\mathbf{K}_{0}+\mathbf{q}}^{\dagger}\left[\bar{\Delta}_{0} i \hat{\sigma}_{y}\right] \hat{c}_{-\mathbf{K}_{0}-\mathbf{q}}^{\dagger}+\text { H.c. },
$$

with $\bar{\Delta}_{0}$ being the order parameter. This is possibly the simplest pairing between the Fermi surfaces $\mathrm{FS}_{ \pm}$enclosing the two nodal points at $\zeta \mathbf{K}_{0}$ and involves the two Weyl quasiparticles with momenta $\mathbf{K}_{0}+\mathbf{q}$ and $-\mathbf{K}_{0}-\mathbf{q}$, where $\pm \mathbf{q}$ lives on the sphere $S_{ \pm}$obtained after shifting $\mathrm{FS}_{ \pm}$by $\mp \mathbf{K}_{0}$ toward the origin. Crucially, the Cooper pair wave function acquires the total Berry flux $4 \pi$ inherited from the parent chiral Weyl fermions [22]. Consequently, its projection onto the sphere $S_{+}$ $\left(S_{-}\right)$features at least one vortex with the unit $(2 \pi)$ vorticity, and the corresponding projected pairing is proportional to a monopole harmonic function $Y_{q, l, m}(\theta, \phi)$, with $4 \pi q$ counting the total Berry flux of the SC state [28].

More formally, the band basis on the Fermi surfaces $\mathrm{FS}_{ \pm}$ is $\hat{\alpha}_{ \pm}^{\dagger}( \pm \mathbf{q})=\sum_{\sigma=\uparrow, \downarrow} \xi_{ \pm, \sigma}( \pm \mathbf{q}) \hat{c}_{ \pm \mathbf{K}_{0} \pm \mathbf{q}, \sigma}^{\dagger}$, with the spinors $\xi_{ \pm, \uparrow}( \pm \mathbf{q})=\left(u_{\mathbf{q}}, v_{\mathbf{q}}\right)^{T}$, chosen so that the Dirac string pierces the sphere at the south pole (spherical polar angle $\theta_{\mathbf{q}}=\pi$ ) since $u_{\mathbf{q}}=\cos \left(\theta_{\mathbf{q}} / 2\right)$ and $v_{\mathbf{q}}=\sin \left(\theta_{\mathbf{q}} / 2\right) e^{i \phi_{\mathbf{q}}}$, and $\phi_{\mathbf{q}}$ is the azimuthal angle. After projecting the pairing Hamiltonian in Eq. (3) onto the $\mathrm{FS}_{ \pm}\left( \pm \mathbf{q} \in \mathrm{FS}_{ \pm}\right)$in the weak-coupling (BCS) regime $\left|\bar{\Delta}_{0}\right| \ll|\mu|$, we obtain

$$
\hat{\tilde{H}}_{\Delta}=\sum_{\mathbf{q}} \hat{\alpha}_{-}^{\dagger}(\mathbf{q}) \tilde{\Delta}(\mathbf{q}) \hat{\alpha}_{+}^{\dagger}(-\mathbf{q})+\text { H.c. }
$$

with the gap function $\tilde{\Delta}(\mathbf{q})=-2 \bar{\Delta}_{0} u_{\mathbf{q}}^{*} v_{\mathbf{q}}^{*}=-\bar{\Delta}_{0}$ $\sin \theta_{\mathbf{q}} e^{-i \phi_{\mathbf{q}}}=-\bar{\Delta}_{0} \sqrt{\frac{4 \pi}{3}} Y_{-1,1,0}\left(\theta_{\mathbf{q}}, \phi_{\mathbf{q}}\right)$, where $Y_{q, l, m}(\theta, \phi)$ is the standard monopole harmonic function [30,31]. Notice that for the monopole pairing in Eq. (4), $2 q=2 \mathcal{C}_{-}=-2$ since $\mathcal{C}_{ \pm} \rightarrow \mathcal{C}_{\mp}$ under $\mathbf{q} \rightarrow-\mathbf{q}$.

In a WSM prone to a superconducting instability, a more conventional intra-FS spin-singlet pairing, which necessarily occurs at a finite momentum $2 \mathbf{K}_{0}$, is also possible and competes with the monopole SC. Furthermore, the inversion symmetry in Weyl materials may be broken, so to account for this effect, we consider slightly different chemical potentials at the two nodes, $\mu_{-}$and $\mu_{+}$, with $|\delta \mu|=\left|\mu_{+}-\mu_{-}\right| \ll \bar{\mu}$, where $\bar{\mu}=\left(\mu_{+}+\mu_{-}\right) / 2$ is the average chemical potential. The mean-field Bogoliubov-de Gennes Hamiltonian that includes both pairing instabilities takes the form

$$
\hat{H}=\sum_{\mathbf{q}} \Psi_{\mathbf{q}}^{\dagger} \hat{H}_{B d G}(\mathbf{q}) \Psi_{\mathbf{q}},
$$

with

$$
\hat{H}_{B d G}(\mathbf{q})=\left[\begin{array}{cccc}
\xi_{\mathbf{q}}^{-} & \Delta_{0} & 0 & \tilde{\Delta}_{\mathbf{q}} \\
\Delta_{0}^{*} & -\xi_{\mathbf{q}}^{-} & \tilde{\Delta}_{\mathbf{q}}^{*} & 0 \\
0 & \tilde{\Delta}_{\mathbf{q}} & \xi_{\mathbf{q}}^{+} & \Delta_{0} \\
\tilde{\Delta}_{\mathbf{q}}^{*} & 0 & \Delta_{0}^{*} & -\xi_{\mathbf{q}}^{+}
\end{array}\right],
$$

and the Nambu basis is $\Psi_{\mathbf{q}}^{\dagger}=\left[\hat{\alpha}_{-}^{\dagger}(\mathbf{q}), \hat{\alpha}_{-}(-\mathbf{q}), \hat{\alpha}_{+}^{\dagger}(\mathbf{q})\right.$, $\left.\hat{\alpha}_{+}(-\mathbf{q})\right]$, while $\xi_{\mathbf{q}}^{ \pm}=v_{F}|\mathbf{q}|-\mu_{ \pm}$. In this basis, we can treat both $s$-wave and $p$-wave intra-FS pairings as long as they separately compete with the monopole SC. Otherwise, the spin index would have to be explicitly restored, and the basis would therefore be doubled.

It is convenient to express the above Hamiltonian by using the $S U(2) \otimes S U(2)$ matrices $\left\{\hat{\tau}_{\alpha} \otimes \hat{\eta}_{\beta}\right\}$, with $\hat{\tau}_{\alpha}$ representing the $S U(2)$ nodal basis, while $\hat{\eta}_{\beta}$ is the particle-hole basis. Here, $\left\{\hat{\tau}_{\alpha}, \hat{\eta}_{\beta}\right\}$ are the Pauli matrices, while $\hat{\tau}_{0}, \hat{\eta}_{0}$ are the $2 \times 2$ unity matrices. Therefore, the Hamiltonian in Eq. (6) in this representation reads

$$
\begin{aligned}
\hat{H}_{B d G}(\mathbf{q})= & \bar{\xi}_{q} \hat{\tau}_{0} \otimes \hat{\eta}_{3}+\frac{\delta \mu}{2} \hat{\tau}_{3} \otimes \hat{\eta}_{3}+\operatorname{Re} \Delta_{0} \hat{\tau}_{0} \otimes \hat{\eta}_{1} \\
& -\operatorname{Im} \Delta_{0} \hat{\tau}_{0} \otimes \hat{\eta}_{2}+\operatorname{Re} \tilde{\Delta}_{\mathbf{q}} \hat{\tau}_{1} \otimes \hat{\eta}_{1}-\operatorname{Im} \tilde{\Delta}_{\mathbf{q}} \hat{\tau}_{1} \otimes \hat{\eta}_{2},
\end{aligned}
$$

where we defined $\bar{\xi}_{\mathbf{q}}=v_{F}|\mathbf{q}|-\bar{\mu}$.

\section{BCS MEAN-FIELD GAP EQUATIONS: CLEAN LIMIT}

The mean-field gap equations for the two competing superconducting orderings are obtained from the finite-temperature Green's function for the effective Bogoliubov-de Gennes Hamiltonian in Eq. (5), which in terms of the valley subblocks reads (see Appendix B)

$$
\hat{\mathcal{G}}_{0}\left(\omega_{n}, \mathbf{q}\right)=\left[-i \omega_{n}+\hat{H}_{B d G}(\mathbf{q})\right]^{-1}=\left[\begin{array}{ll}
\hat{G}_{0}^{--} & \hat{G}_{0}^{-+} \\
\hat{G}_{0}^{+-} & \hat{G}_{0}^{++}
\end{array}\right],
$$

where $\hat{G}_{0}^{\rho \zeta}, \rho, \zeta= \pm$ are the $2 \times 2$ submatrices and $\omega_{n}=$ $(2 n+1) \pi T$ is the fermionic Matsubara frequency at temperature $T$. The gap equations for the conventional intra-Fermi surface pairing and the monopole SC then can be compactly written as

$$
\Delta_{\eta}(\mathbf{q})=-T \sum_{\mathbf{q}, \omega_{n}} V_{\eta}\left(\mathbf{q}, \mathbf{q}^{\prime}\right)\left\langle\hat{\alpha}_{-}\left(\mathbf{q}^{\prime}\right) \hat{\alpha}_{\zeta}\left(-\mathbf{q}^{\prime}\right)\right\rangle,
$$

where $\zeta=-(\zeta=+)$ for $\eta=$ intra $(\eta=$ inter $)$ corresponding to the spherical (monopole) harmonic pairing, and $V_{\eta}$ are the pairing potentials. In terms of the Green's function in Eq. (8), $\left\langle\hat{\alpha}_{-}(\mathbf{q}) \hat{\alpha}_{\zeta}(-\mathbf{q})\right\rangle=\left[\hat{G}_{0}^{-\zeta}\right]_{21}$. The explicit form of the gap equations is given by Eqs. (C12) and (C13), from which we can conclude that when these two superconducting orders compete, new instabilities can be generated, but in the insulating (particle-hole) channels. More specifically, when the intra-FS pairing is $s$ wave, the two $p$-wave charge density wave orders in the $x$ and $y$ directions may get generated. This is so when the two superconducting orders coexist, which is, indeed, possible, unless the two orders exhibit the same $\theta$-dependent form factors, as we show below. The study of the effects of a generated insulating state on the superconducting instabilities is, however, beyond the scope of the current work.

The pairing potentials for the spherical harmonics and the monopole channels when $\mu_{+}=\mu_{-}=\mu$, dictated by the form of the corresponding pairing functions, are, in general, given by

$$
\begin{aligned}
& V_{\text {intra }}\left(\mathbf{q}, \mathbf{q}^{\prime}\right)=V_{0} Y_{l, m}\left(\theta_{\mathbf{q}}, \phi_{\mathbf{q}}\right) Y_{l, m}^{*}\left(\theta_{\mathbf{q}^{\prime}}, \phi_{\mathbf{q}^{\prime}}\right), \\
& V_{\text {inter }}\left(\mathbf{q}, \mathbf{q}^{\prime}\right)=\tilde{V}_{0} Y_{q, j, m}\left(\theta_{\mathbf{q}}, \phi_{\mathbf{q}}\right) Y_{q, j, m}^{*}\left(\theta_{\mathbf{q}^{\prime}}, \phi_{\mathbf{q}^{\prime}}\right),
\end{aligned}
$$

where $Y_{l, m}(\theta, \phi)=f_{l}(\theta) e^{i m \phi}$ and $Y_{q, j, m}=e^{i(m+q) \phi} g(\theta)$, where $Y_{q, j, m}(\theta, \phi)$ is the monopole harmonic with the form given by Eq. (18). 
Let us now consider the $T=0$ BCS gap equation for a more general intra-FS pairing as defined above. For convenience, we introduce the notation

$$
\begin{aligned}
& \Delta_{\text {inter }}(\mathbf{q})=\bar{\Delta}_{0} d_{\text {inter }}(\theta) e^{i(m+q) \phi}, \\
& \Delta_{\text {intra }}(\mathbf{q})=\Delta_{l m, 0} d_{\text {intra }}(\theta) e^{i m^{\prime} \phi} .
\end{aligned}
$$

The corresponding generalized zero-temperature BCS gap equations, derived in Appendix C, read

$$
\lambda_{\eta}^{-1}=\int \frac{d \theta d \phi}{4 \pi} \sin \theta\left|d_{\eta}(\theta)\right|^{2}\left[2 \ln \left(2 \omega_{D}\right)-\frac{1}{2} \sum_{s= \pm} \ln \left(A_{+}+2 s B_{q}\right)\right],
$$

with $\lambda_{\eta}(\eta=$ inter, intra) as the effective coupling constants, given by

$$
\lambda_{\text {inter }}=V_{0} \rho(\mu), \quad \lambda_{\text {intra }}=\frac{\tilde{V}_{0} \rho(\mu)}{4 \pi} \int_{0}^{2 \pi} d \phi \int_{0}^{\pi} d \theta \sin \theta\left|Y_{-1,1,0}(\theta, \phi)\right|^{2}
$$

[see also Eq. (C25) and discussion therein]. Here, we defined the coefficients

$$
\begin{aligned}
A_{ \pm} & =\Delta_{l m, 0}^{2} f_{l}^{2}(\theta) \pm \bar{\Delta}_{0}^{2} g^{2}(\theta), \\
2 B_{q} & =2\left[\operatorname{Re} \Delta_{\text {inter }}(\mathbf{q}) \operatorname{Re} \Delta_{\text {intra }}(\mathbf{q})+\operatorname{Im} \Delta_{\text {inter }}(\mathbf{q}) \operatorname{Im} \Delta_{\text {intra }}(\mathbf{q})\right] \\
& =2 \Delta_{l m, 0} \bar{\Delta}_{0} g(\theta) f_{l}(\theta) \cos \left\{\left[m^{\prime}-(m+q)\right] \phi\right\} \equiv B \cos \left\{\left[m^{\prime}-(m+q)\right] \phi\right\} .
\end{aligned}
$$

The integral over the azimuthal angle is calculated as follows after setting $r=m^{\prime}-(m+q)$ :

$$
\begin{aligned}
\int_{0}^{2 \pi} d \phi \ln \left[A_{+} \pm B \cos (r \phi)\right] & =\sum_{n=1}^{r} \int_{2 \pi(n-1) / r}^{2 \pi n / r} d \phi \ln \left[A_{+} \pm B \cos (r \phi)\right] \\
& =\frac{1}{r} \sum_{n=1}^{r} \int_{2 \pi(n-1)}^{2 \pi n} \ln \left[A_{+} \pm B \cos \phi\right]=2 \pi \ln \left[\frac{1}{2}\left(A_{+}+\sqrt{A_{+}^{2}-B^{2}}\right)\right] \\
& =2 \pi \ln \left[\frac{1}{2}\left(A_{+}+\left|A_{-}\right|\right)\right] .
\end{aligned}
$$

Remarkably, when $\left|f_{l}(\theta)\right| \sim|g(\theta)|$, i.e., $\left|f_{l}(\theta)\right|$ and $|g(\theta)|$ are the same functions up to a real coefficient, and $m^{\prime} \neq m+$ $q$, Eq. (15) determines the competition between a monopole SC phase and a conventional spherical harmonic SC phase since, in that case,

$$
A_{+}+\left|A_{-}\right|=\left\{\begin{array}{cc}
2 \bar{\Delta}_{0}^{2} g^{2}(\theta), & \bar{\Delta}_{0}>\Delta_{l m, 0}, \\
2 \Delta_{l m, 0}^{2} f_{l}^{2}(\theta), & \bar{\Delta}_{0}<\Delta_{l m, 0} .
\end{array}\right.
$$

Therefore, we conclude that in this case a sharp boundary exists between the monopole SC phase and the spherical harmonic $Y_{l, m^{\prime}}(\theta, \phi)$ pairing, where the stronger coupling dominates. Analogously, also when $\left|f_{l}(\theta)\right| \sim|g(\theta)|$ and $m^{\prime}=$ $m+q$, the gap equations (12) imply that the coexistence is possible only when the two couplings are equal. Namely, in that case, the two equations reduce to

$$
\lambda_{\eta}^{-1}=\int d \theta \sin \theta\left|f_{l}(\theta)\right|^{2} \ln \left[\frac{2 \omega_{D}}{f_{l}^{2}(\theta)\left|\Delta_{l m, 0}^{2}-\bar{\Delta}_{0}^{2}\right|}\right]
$$

for $\eta=$ intra, inter. Therefore, provided that $\left|f_{l}(\theta)\right| \sim|g(\theta)|$, $m^{\prime}>0$, and $m+q>0$, the phases repel each other. As we show below, the last two conditions can be removed because of the gauge freedom in choosing where the Dirac string pierces the Fermi sphere. In other words, a spherical harmonic and the monopole $\mathrm{SC}$ repel each other when the corresponding $\theta$-dependent form factors satisfy $\left|f_{l}(\theta)\right| \sim|g(\theta)|$. It turns out that for any monopole harmonic [see Eq. (18)] $Y_{-q, q, 0}(\theta, \phi) \sim$ $(\sin \theta)^{q} e^{i q \phi}$ and spherical harmonic $Y_{q, q}(\theta, \phi) \sim(\sin \theta)^{q} e^{i q \phi}$, the corresponding pairings always repel each other, and we name this mechanism topological repulsion.

To show the gauge independence of this principle, we recall that gauge choices where the Dirac string originating from the monopole goes through the north pole $(\theta=$ $0)$ and the south pole $(\theta=\pi)$ are related by a coordinate transformation $\theta \rightarrow \pi-\theta$ and $\phi \rightarrow-\phi$. The equivalent gauge classes are given by $Y_{q, j, m}$ and $Y_{-q, j,-m}$, which can be shown as follows. We start from the form of the monopole harmonic [28]

$$
Y_{q, j, m}(\theta, \phi)=\sqrt{\frac{2 j+1}{4 \pi}} e^{i(m+q) \phi} d_{m,-q}^{j}(\theta),
$$

where

$$
\begin{aligned}
d_{m, l}^{j}(\theta)= & \sqrt{\frac{(j+l) !(j-l) !}{(j+m) !(j-m) !}}\left(\cos \frac{\theta}{2}\right)^{l+m}\left(\sin \frac{\theta}{2}\right)^{l-m} \\
& \times P_{j-l}^{l-m, l+m}(\cos \theta)
\end{aligned}
$$

and the function $P_{n}^{a, b}(x)$ is defined as

$$
\begin{aligned}
P_{n}^{a, b}(x)= & \frac{(-1)^{n}}{2^{n} n !}(1-x)^{-a}(1+x)^{-b} \\
& \times \frac{d^{n}}{d x^{n}}\left[(1-x)^{a+n}(1+x)^{b+n}\right] .
\end{aligned}
$$


Then, in the next step one can easily see that

$$
d_{m, l}^{j}(\theta) \rightarrow d_{m, l}^{j}(\pi-\theta)=(-1)^{j-l} d_{-m, l}^{j}(\theta),
$$

and under $\phi \rightarrow-\phi$

$$
e^{i(q+m) \phi} \rightarrow e^{i(-q-m) \phi} .
$$

The last two equations, together with the form of the monopole harmonic functions in Eq. (20), show that the monopole harmonics $Y_{q, j, m}(\theta, \phi)$ and $Y_{-q, j,-m}(\theta, \phi)$ are equivalent with respect to the choice of the direction of the Dirac string. Therefore, the topological repulsion is operative irrespective of the sign of $m+q$ in the monopole harmonic $Y_{q, l, m}(\theta, \phi)$ [see Eq. (18)].
We now discuss special cases when the $s$-wave and $p$-wave pairings compete with the monopole $\operatorname{SC} Y_{-1,1,0}(\theta, \phi)$ (the Dirac string pierces the south pole).

\section{A. Monopole versus $s$-wave pairing state}

The above criterion implies that the coexistence of the $s$ wave pairing and the monopole harmonic $Y_{-1,1,0}(\theta, \phi)$ may be possible, as indeed displayed in Fig. 1(a). This conclusion is based on the solution of the self-consistent gap equations, which read

$$
\begin{aligned}
& \lambda_{\text {intra }}^{-1}=2 \pi \int_{0}^{\pi} \frac{d \theta}{4 \pi} \sin \theta\left\{2 \ln \left(2 \omega_{D}\right)-\ln \left[\frac{1}{2}\left(\Delta_{s}^{2}+\bar{\Delta}_{0}^{2} \sin ^{2} \theta+\left|\Delta_{s}^{2}-\bar{\Delta}_{0}^{2} \sin ^{2} \theta\right|\right)\right]\right\}=2 \ln \left(2 \omega_{D}\right)-\frac{1}{2} \mathcal{J}^{(1)}, \\
& \lambda_{\text {inter }}^{-1}=2 \pi \int_{0}^{\pi} \frac{d \theta}{4 \pi} \sin ^{3} \theta\left\{2 \ln \left(2 \omega_{D}\right)-\ln \left[\frac{1}{2}\left(\Delta_{s}^{2}+\bar{\Delta}_{0}^{2} \sin ^{2} \theta+\left|\Delta_{s}^{2}-\bar{\Delta}_{0}^{2} \sin ^{2} \theta\right|\right)\right]\right\}=\frac{4}{3} \ln \left(2 \omega_{D}\right)-\frac{1}{2} \mathcal{J}^{(3)} .
\end{aligned}
$$

Here, we defined the integrals (for $n=0,1$ )

$$
\begin{aligned}
\mathcal{J}^{(2 n+1)}= & \int_{0}^{\pi} d \theta \sin ^{2 n+1} \theta \\
& \times \ln \left[\frac{1}{2}\left(\Delta_{s}^{2}+\bar{\Delta}_{0}^{2} \sin ^{2} \theta+\left|\Delta_{s}^{2}-\bar{\Delta}_{0}^{2} \sin ^{2} \theta\right|\right)\right] .
\end{aligned}
$$

To evaluate these angular integrals, one needs to distinguish two separate regimes in the parameter space in order to handle correctly the absolute value in the integrand. For $\Delta_{s}>\bar{\Delta}_{0}$, it is straightforward to obtain (for $n=0,1$ )

$$
\mathcal{J}^{(2 n+1)}=\frac{2^{n+1} n !}{(2 n+1) ! !} \ln \left(\Delta_{s}^{2}\right) .
$$

On the other hand, for $\Delta_{s}<\bar{\Delta}_{0}$, we define the angular parameter $\sin \theta_{0}=\Delta_{s} / \bar{\Delta}_{0}$, which allows us to calculate the integrals by splitting the domain into the subintervals $\theta \in$ $\left[0, \theta_{0}\right] \cup\left[\theta_{0}, \pi-\theta_{0}\right] \cup\left[\pi-\theta_{0}, \pi\right]$. The corresponding result is $\left(\Delta_{s}<\bar{\Delta}_{0}\right)$

$$
\begin{aligned}
\mathcal{J}^{(1)}= & 2 \ln \left(\Delta_{s}^{2}\right)+4\left(\ln \left[\frac{1+\cos \theta_{0}}{\sin \theta_{0}}\right]-\cos \theta_{0}\right) \\
\mathcal{J}^{(3)}= & \frac{4}{3} \ln \left(\Delta_{s}^{2}\right)-\frac{1}{3}\left(8-9 \cos \theta_{0}+\cos 3 \theta_{0}\right) \\
& +\frac{1}{9}\left\{24 \ln \left[\frac{1+\cos \theta_{0}}{\sin \theta_{0}}\right]+\cos 3 \theta_{0}\left(1-3 \ln \sin \theta_{0}\right)\right. \\
& \left.+3 \cos \theta_{0}\left(-7+9 \ln \sin \theta_{0}\right)\right\}
\end{aligned}
$$

To understand the phase diagram displayed in Fig. 1(a), we start from $\lambda_{\text {inter }}=0$ and thus $\Delta_{0}=0$ and find that for any $\lambda_{\text {inter }}<3 \lambda_{s} / 2$, the system is in the pure $s$-wave state. The line at which the coexistence of these two SC phases sets in is given by the condition $\Delta_{s}=\bar{\Delta}_{0}\left(\theta_{0}=\pi / 2\right)$; the form is obtained by using Eqs. (23) and (26) and reads

$$
\lambda_{\text {inter }}^{-1}=\frac{4}{3} \ln \left(2 \omega_{D}\right)-\frac{2}{3} \ln \left(\Delta_{0}^{2}\right)=\frac{2}{3} \lambda_{\text {intra }}^{-1} ;
$$

see also additional analysis on the coexistence across this line in Appendix D. The transition from the pure $s$ wave to the mixed SC is discontinuous since the solution $\Delta_{s} \neq 0, \Delta_{0}=0$ is valid up to the phase boundary line [the blue solid line in Fig. 1(a)], and it jumps to $\Delta_{s}=\Delta_{0} \neq 0$. As the coupling $\lambda_{\text {intra }}$ is further increased across this line, the coexistence regime persists until the pure monopole SC state is reached, which occurs for $\Delta_{s}=0$. Using Eq. (23), we obtain the form of the phase boundary between the hybrid SC and the pure monopole SC [the red solid line in Fig. 1(b)]:

$$
\lambda_{\text {inter }}^{-1}-\frac{2}{3} \lambda_{\text {intra }}^{-1}=-\frac{2}{9} .
$$

The phase transition from the coexisting region to the pure monopole state is discontinuous, as can be readily shown by inserting the form of the phase boundary given by Eq. (28) into Eqs. (23) and (26), valid for $0 \neq \Delta_{s}<\Delta_{0}$, which shows that as the boundary is approached from the coexistence region, $\Delta_{s}$ cannot vanish. Finally, we point out that the obtained phase diagram, shown in Fig. 1(a), is qualitatively similar to the one for the mixed $s+i d$ superconductor in a twodimensional Fermi liquid [32].

To include the effect of the inversion symmetry breaking, we take different chemical potentials at the two nodes and consider the system close to the critical temperature $T_{c}$. In the symmetric case, $\mu=0$, the finite-temperature phase boundary is given by the condition $\lambda_{\text {intra }}=\lambda_{\text {inter }}$. When the inversion symmetry is broken, the value of the effective inter-FS pairing potential at the phase boundary, however, increases, implying that the intra-FS $s$-wave superconductor becomes more favorable, as shown in Fig. 2.

\section{B. Monopole versus $\boldsymbol{p}$-wave pairing state}

Let us now consider the $p$-wave case $p_{ \pm}=p_{x} \pm i p_{y}$ and $p_{z}$, defined by $\Delta_{p_{z}}=\Delta_{p_{z}, 0} \cos \theta, \Delta_{p_{ \pm}}=\Delta_{p_{ \pm}, 0} e^{ \pm i \phi} \sin \theta$. For $p_{z}$, we obtain the same behavior as for the $s$-wave case because this harmonic is independent of the azimuthal angle $\phi$, while the monopole harmonic is $\phi$ dependent, and the corresponding 
$\theta$-dependent form factors are different. On the other hand, using Eq. (17), for both $p_{ \pm}$pairings we arrive at the BCS equation

$$
\begin{aligned}
\lambda_{p_{ \pm}}^{-1}= & 2 \pi \int_{0}^{\pi} \frac{d \theta}{4 \pi} \sin ^{3} \theta\left[2 \ln \left(2 \omega_{D}\right)-2 \ln (\sin \theta)\right. \\
& \left.-2 \ln \left|\Delta_{p_{ \pm}, 0}^{2}-\bar{\Delta}_{0}^{2}\right|\right] \\
= & \frac{8}{3} \ln \left(2 \omega_{D}\right)-\frac{5}{9}(5-\ln 64)-\ln \left|\Delta_{p_{ \pm}, 0}^{2}-\bar{\Delta}_{0}^{2}\right|, \\
\lambda_{\text {intra }}^{-1}= & \frac{8}{3} \ln \left(2 \omega_{D}\right)-\frac{5}{9}(5-\ln 64)-\ln \left|\Delta_{p_{ \pm}, 0}^{2}-\bar{\Delta}_{0}^{2}\right| .
\end{aligned}
$$

In this case, coexistence will arise only for $\lambda_{p_{ \pm}}=\lambda_{\text {intra }}$, as shown in Fig. 1(b). Otherwise, the dominant phase will correspond to a larger coupling. After defining the effective coupling by $\lambda_{\text {eff }} \equiv \lambda_{p_{ \pm}}=\lambda_{\text {intra }}$ and solving the above selfconsistent gap equations, we obtain

$$
\left|\Delta_{p_{ \pm}, 0}^{2}-\bar{\Delta}_{0}^{2}\right|=2 \omega_{D} e^{-\frac{1}{12}(5-\ln 64)} e^{-\frac{3}{8} \lambda_{\text {eff }}^{-1}} .
$$

Ultimately, even in this fine-tuned situation, when fluctuation effects are accounted for, we expect that the phases repel each other due to their incompatible topological structures: while the monopole SC features a double vortex coming from individual Fermi surfaces $\mathrm{FS}_{ \pm}$, the $p$-wave harmonic picks up a vortex-antivortex pair at each of them.

\section{IMPURITY SCATTERING}

Let us now consider the effect of scattering by randomly distributed, nonmagnetic impurities, with a concentration $n_{\text {imp }}$, on the superconducting instabilities. Within the first Born approximation, this can be captured through an averaged self-energy matrix of the form [33]

$$
\hat{\Sigma}\left(\omega_{n}, \mathbf{q}\right)=n_{\text {imp }} \sum_{\mathbf{q}_{1}} \hat{W}_{\mathbf{q}, \mathbf{q}_{1}} \hat{\mathcal{G}}\left(\omega_{n}, \mathbf{q}_{1}\right) \hat{W}_{\mathbf{q}_{1}, \mathbf{q}},
$$

where $\hat{G}\left(\omega_{n}, \mathbf{q}\right)$ is the fully dressed Green's function matrix, arising from the solution of the Dyson equation

$$
\hat{\mathcal{G}}^{-1}\left(\omega_{n}, \mathbf{q}\right)=\hat{\mathcal{G}}_{0}^{-1}\left(\omega_{n}, \mathbf{q}\right)-\hat{\Sigma}\left(\omega_{n}, \mathbf{q}\right) .
$$

We assume, for simplicity, that the scattering potential matrix is momentum independent and reads

$$
\hat{W}_{\mathbf{q}, \mathbf{q}_{1}}=u \hat{\tau}_{1} \otimes \hat{\eta}_{0}+v \hat{\tau}_{0} \otimes \hat{\eta}_{3},
$$

where $u$ and $v$ are, respectively, the inter- and intra-FS scattering strengths and we assume a Gaussian disorder distribution. We solve the Dyson equation by taking the ansatz for the fully dressed Green's function so that it retains the same structure as that without the disorder, but with renormalized parameters $\omega_{n, R}, \bar{\Delta}(\mathbf{q})_{R}, \bar{\Delta}_{0, R}$, and $\bar{\xi}_{n, q, R}$. In the following, we neglect the asymmetry between the chemical potentials at the two Weyl points and thus set $\delta \mu=0$. To leading order in the impurity strength, we obtain the renormalized parameters in the form (see Appendix E for details)

$$
\begin{aligned}
& \omega_{n, R}=\omega_{n}\left[1+\frac{1}{2}\left(\tau_{\text {intra }}^{-1}+\tau_{\text {inter }}^{-1}\right)\left|\omega_{n}\right|^{-1}\right], \\
& \Delta_{0, R}=\Delta_{0}\left[1+\frac{1}{2}\left(\tau_{\text {intra }}^{-1}-\tau_{\text {inter }}^{-1}\right)\left|\omega_{n}\right|^{-1}\right],
\end{aligned}
$$

while the band dispersion and the monopole pairing gap function remain unrenormalized. Here, we define the inverse inter-FS scattering time $\tau_{\text {inter }}^{-1}=2 \pi n_{\text {imp }} u^{2} \rho(\mu)$, with analogous notation for the intra-FS scattering.

The gap equation for the $s$-wave pairing in the weakcoupling limit, $\omega_{D} \gg T_{c}$, then reads

$$
\frac{T_{c}}{\lambda_{\text {intra }}}=\ln \left(\frac{\Gamma_{c}}{2 \pi T_{c}}\right)-\psi\left(\frac{1}{2}+\frac{\tau_{\text {inter }}^{-1}}{2 \pi T_{c}}\right),
$$

where $\Gamma_{c}$ is an upper cutoff for the Matsubara frequency sum and $\psi(x)$ is the digamma function. When only the intra-FS scattering is present, the critical temperature remains unchanged, consistent with Anderson's theorem [34] and its generalized version for unconventional pairing states in terms of the superconducting fitness $[35,36]$. Notice that the density wave nature of this superconducting order does not play a role since the disorder preserves translational symmetry on average.

For the monopole superconductor, on the other hand, because of the form of its projection on the FS, the gap does not renormalize. Consequently, the effect of both intra- and inter-FS disorders is to lower its critical temperature,

$$
\frac{T_{c}}{\lambda_{\text {inter }}}=\ln \left(\frac{\Gamma_{c}}{2 \pi T_{c}}\right)-\psi\left(\frac{1}{2}+\frac{\tau_{\text {intra }}^{-1}+\tau_{\text {inter }}^{-1}}{4 \pi T_{c}}\right) .
$$

Notice that both types of disorder anticommute with the pairing matrix for the monopole $\mathrm{SC}$, as can be directly checked from Eq. (6). Therefore, the superconducting fitness function is nonvanishing for either of them, consistent with the correction to $T_{c}$ given by Eq. (36).

To illustrate the competition of the two superconducting phases when the intra-FS scattering is turned on, we plot the ratio between the inter- and intra-FS pairing interactions, $\delta \lambda=\lambda_{\text {inter }} / \lambda_{\text {intra }}-1$, at the phase boundary (the two critical temperatures are equal) as a function of the intra-FS inverse scattering time, shown in Fig. 3. The intra-FS scattering suppresses the monopole $\mathrm{SC}$ since as this scattering increases, the phase boundary moves toward larger values of the inter-FS pairing strength. A similar behavior is observed when the inter-FS scattering is tuned for a fixed intra-FS disorder.

\section{DISCUSSION AND OUTLOOK}

To summarize, we here demonstrated that the monopole and conventional spherical harmonic SCs quite generically coexist, while the repulsion can take place when the absolute value of the monopole charge matches the angular momentum quantum number of the spherical harmonic. We illustrated this general principle with the particular examples of finite-momentum $s$ - and $p$-wave pairings competing with the monopole SC, which, respectively, coexist and repel. We showed that the $s$-wave pairing is more stable both for unequal chemical potentials at the nodes and in the presence of pointlike charged impurities. Close to the phase boundary, the system features gapless modes at the interface of the topologically nontrivial monopole harmonic and the trivial $s$-wave superconducting domains, providing an experimental signature of the monopole SC.

In spite of many realized Weyl metals, the signatures of the Weyl superconductivity were only recently reported in $\mathrm{UTe}_{2}$ 
[37]. Particularly relevant in this context is the observation that the superconducting state is a time-reversal symmetrybreaking two-component spin-triplet order parameter, which as such may feature a monopole component, but the nature of the order parameter is still an open question.

Our work should motivate further studies of the monopole harmonic SCs, such as their competition with the insulating instabilities, particularly with those displaying the monopole structure [38]. Finally, observable consequences of these exotic states beyond the surface Majorana modes are yet to be explored, for instance, impurity resonances [39].

\section{ACKNOWLEDGMENTS}

We thank B. Roy for insightful comments. This work was supported by Fondecyt Grants No. 1190361 and No. 1200399 and by ANID PIA/Anillo Grant No. ACT192023. V.J. acknowledges the support of the Swedish Research Council (Grant No. VR 2019-04735).

\section{APPENDIX A: DETAILS OF THE MODEL}

We start by considering the model introduced in Refs. [28,29], which describes the mean-field Cooper pairing between quasiparticle excitations at two Fermi surfaces (FSs) enclosing the two nodal points in a Weyl semimetal. For this model, the effective Hamiltonian is

$$
\hat{H}=\hat{H}_{\text {Weyl }}+\hat{H}_{\Delta} .
$$

Here, $\hat{H}_{\text {Weyl }}=\sum_{\mathbf{k}} \hat{c}_{\mathbf{k}}^{\dagger} h(\mathbf{k}) \hat{c}_{\mathbf{k}}$, where the matrix

$$
\begin{aligned}
h(\mathbf{k})= & t \sin k_{x} \hat{\sigma}_{x}+t \sin k_{y} \hat{\sigma}_{y} \\
& +t\left(2-\cos k_{x}-\cos k_{y}-\cos k_{z}+\cos K_{0}\right) \hat{\sigma}_{z}-\mu
\end{aligned}
$$

and $\mu>0$ is the chemical potential.

The band structure in Eq. (A2) possesses two Weyl nodes along the $k_{z}$ direction, given by $\zeta \mathbf{K}_{0}=\left(0,0, \zeta K_{0}\right)$, each with opposite topological charge $\mathcal{C}_{\zeta}=\zeta= \pm 1$. We consider only isotropic nodes by choosing $t=v_{F}$ and $K_{0}=\pi / 2$. Therefore, we write the effective Hamiltonian describing Weyl quasiparticles in the vicinity of each node $\zeta \mathbf{K}_{0}$ as $\hat{H}_{\text {Weyl }}=$ $\sum_{\zeta= \pm, \mathbf{q}} \hat{c}_{\zeta \mathbf{K}_{0}+\mathbf{q}}^{\dagger} \hat{h}_{\zeta}(\mathbf{q}) \hat{c}_{\zeta \mathbf{K}_{0}+\mathbf{q}}$, with

$$
\hat{h}_{\zeta}(\mathbf{q})=v_{F}\left(\hat{\sigma}_{x} q_{x}+\hat{\sigma}_{y} q_{y}+\zeta \hat{\sigma}_{z} q_{z}\right)-\mu,
$$

which we use in the main text.

\section{APPENDIX B: BOGOLIUBOV-DE GENNES FINITE-TEMPERATURE FIELD THEORY}

From the effective mean-field Bogoliubov-de Gennes Hamiltonian in Eq. (6), we can construct a finite-temperature field theory in terms of the Grassmann four-component fermion fields in the Nambu bases $\Psi_{\mathbf{q}}$ and $\Psi_{\mathbf{q}}^{\dagger}$. The corresponding partition function is given by the functional integral

$$
Z_{0}=\int \mathcal{D} \Psi_{\mathbf{q}}^{\dagger} \mathcal{D} \Psi_{\mathbf{q}} e^{-S\left[\Psi_{\mathbf{q}}^{\dagger}, \Psi_{\mathbf{q}}\right]},
$$

with the action in compactified Euclidean time $0 \leqslant \tau \leqslant \beta$ (for $\beta=1 / T)$,

$$
S\left[\Psi_{\mathbf{q}}^{\dagger}, \Psi_{\mathbf{q}}\right]=\int_{0}^{\beta} d \tau \Psi_{\mathbf{q}}^{\dagger}\left[\hat{\tau}_{0} \otimes \hat{\eta}_{0} \frac{\partial}{\partial \tau}+\hat{H}_{B d G}(\mathbf{q})\right] \Psi_{\mathbf{q}} .
$$

Therefore, the corresponding matrix Green's function satisfies the differential equation

$$
\left[\frac{\partial}{\partial \tau}+\hat{H}_{B d G}(\mathbf{q})\right] \hat{\mathcal{G}}_{0}(\tau, \mathbf{q})=\delta(\tau),
$$

which in the Matsubara frequency space assumes the form

$$
\hat{\mathcal{G}}_{0}\left(\omega_{n}, \mathbf{q}\right)=\int_{0}^{\beta} d \tau e^{i \omega_{n} \tau} \hat{\mathcal{G}}_{0}(\tau, \mathbf{q})
$$

with $\omega_{n}=(2 n+1) \pi / \beta$ for $n \in \mathbb{Z}$. We now solve Eq. (B3) to obtain

$$
\hat{\mathcal{G}}_{0}\left(\omega_{n}, \mathbf{q}\right)=\left[-i \omega_{n} \hat{\tau}_{0} \otimes \hat{\eta}_{0}+\hat{H}_{B d G}(\mathbf{q})\right]^{-1} .
$$

\section{APPENDIX C: CLEAN LIMIT}

We first analyze the system in the absence of impurities to find the critical temperature in this clean limit. We first rewrite the Hamiltonian in Eq. (7) in terms of the $16 \Gamma$ matrices, $\Gamma_{i j}=$ $\hat{\tau}_{i} \otimes \hat{\eta}_{j}, i, j=0,1,2,3$,

$$
\hat{H}_{B d G}(\mathbf{q})=\sum_{\alpha=1}^{3} a_{0 \alpha} \Gamma_{0 \alpha}+a_{11} \Gamma_{11}+a_{12} \Gamma_{12}+a_{33} \Gamma_{33},
$$

where we define the coefficients

$$
\begin{aligned}
& a_{03}=\bar{\xi}_{q}, \quad a_{01}=\operatorname{Re} \Delta_{0}, \quad a_{02}=-\operatorname{Im} \Delta_{0}, \\
& a_{11}=\operatorname{Re} \tilde{\Delta}(\mathbf{q}), \quad a_{12}=-\operatorname{Im} \tilde{\Delta}(\mathbf{q}), \quad a_{33}=\frac{\delta \mu}{2} .
\end{aligned}
$$

The Green's function is obtained by calculating the inverse matrix in Eq. (B5) as follows:

$$
\hat{\mathcal{G}}_{0}\left(\omega_{n}, \mathbf{q}\right)=\left[i \omega_{n} \hat{\tau}_{0} \otimes \hat{\eta}_{0}+\hat{H}_{B d G}(\mathbf{q})\right]\left[\omega_{n}^{2}+\hat{H}_{B d G}(\mathbf{q})^{2}\right]^{-1}
$$

From Eq. (C1) and using the anticommutation relations of the Pauli matrices, we obtain

$$
\begin{aligned}
\hat{H}_{B d G}(\mathbf{q})^{2}= & \Gamma_{00} b^{2}+2\left(a_{01} a_{11} \Gamma_{01} \Gamma_{11}+a_{02} a_{12} \Gamma_{02} \Gamma_{12}\right. \\
& \left.+a_{03} a_{33} \Gamma_{03} \Gamma_{33}\right)
\end{aligned}
$$

where

$$
b^{2}=\sum_{\alpha=1}^{3} a_{0 \alpha}^{2}+a_{11}^{2}+a_{12}^{2}+a_{33}^{2} .
$$

After some straightforward algebra, we obtain the final result:

$$
\hat{\mathcal{G}}_{0}\left(\omega_{n}, \mathbf{q}\right)=i \omega_{n} \frac{\bar{E}_{q}^{2} \Gamma_{00}-2 B_{q} \Gamma_{10}+\delta \mu \bar{\xi}_{q} \Gamma_{30}}{\bar{E}_{q}^{4}-4 B_{q}^{2}-\delta \mu^{2} \bar{\xi}_{q}^{2}}+\sum_{j=1}^{12} \hat{A}_{j} .
$$

Here, we define the parameters

$$
\begin{aligned}
& \bar{E}_{q}^{2}=\bar{\xi}_{q}^{2}+\left|\Delta_{0}\right|^{2}+|\tilde{\Delta}(\mathbf{q})|^{2}+\frac{\delta \mu^{2}}{4}+\omega_{n}^{2}, \\
& B_{q}=\operatorname{Re} \Delta_{0} \operatorname{Re} \tilde{\Delta}(\mathbf{q})+\operatorname{Im} \Delta_{0} \operatorname{Im} \tilde{\Delta}(\mathbf{q})
\end{aligned}
$$


and the set of matrices

$$
\begin{aligned}
& \hat{A_{1}}=-\frac{\bar{\xi}_{q} \bar{E}_{q}^{2}+\bar{\xi}_{q} \delta \mu^{2}}{\bar{E}_{q}^{4}-4 B_{q}^{2}-\delta \mu^{2} \bar{\xi}_{q}^{2}} \hat{\tau}_{0} \otimes \hat{\eta}_{3} \equiv \tilde{a}_{03} \Gamma_{03}, \\
& \hat{A}_{2}=\frac{2 B_{q} \operatorname{Re} \tilde{\Delta}(\mathbf{q})-\bar{E}_{q}^{2} \operatorname{Re} \Delta_{0}}{\bar{E}_{q}^{4}-4 B_{q}^{2}-\delta \mu^{2} \bar{\xi}_{q}^{2}} \hat{\tau}_{0} \otimes \hat{\eta}_{1} \equiv \tilde{a}_{01} \Gamma_{01}, \\
& \hat{A}_{3}=\frac{\bar{E}_{q}^{2} \operatorname{Im} \Delta_{0}-2 B_{q} \operatorname{Im} \tilde{\Delta}(\mathbf{q})}{\bar{E}_{q}^{4}-4 B_{q}^{2}-\delta \mu^{2} \bar{\xi}_{q}^{2}} \hat{\tau}_{0} \otimes \hat{\eta}_{2} \equiv \tilde{a}_{02} \Gamma_{02}, \\
& \hat{A}_{4}=\frac{2 B_{q} \operatorname{Re} \Delta_{0}-\bar{E}_{q}^{2} \operatorname{Re} \tilde{\Delta}(\mathbf{q})}{\bar{E}_{q}^{4}-4 B_{q}^{2}-\delta \mu^{2} \bar{\xi}_{q}^{2}} \hat{\tau}_{1} \otimes \hat{\eta}_{1} \equiv \tilde{a}_{11} \Gamma_{11}, \\
& \hat{A}_{5}=\frac{\bar{E}_{q}^{2} \operatorname{Im} \tilde{\Delta}(\mathbf{q})-2 B_{q} \operatorname{Im} \Delta_{0}}{\bar{E}_{q}^{4}-4 B_{q}^{2}-\delta \mu^{2} \bar{\xi}_{q}^{2}} \hat{\tau}_{1} \otimes \hat{\eta}_{2} \equiv \tilde{a}_{12} \Gamma_{12}, \\
& \hat{A}_{6}=\frac{2 B_{q} \bar{\xi}_{q}}{\bar{E}_{q}^{4}-4 B_{q}^{2}-\delta \mu^{2} \bar{\xi}_{q}^{2}} \hat{\tau}_{1} \otimes \hat{\eta}_{3} \equiv \tilde{a}_{13} \Gamma_{13}, \\
& \hat{A}_{7}=-\frac{\delta \mu\left(\bar{\xi}_{q}^{2}+\bar{E}_{q}^{2}\right)}{\bar{E}_{q}^{4}-4 B_{q}^{2}-\delta \mu^{2} \bar{\xi}_{q}^{2}} \hat{\tau}_{3} \otimes \hat{\eta}_{3} \equiv \tilde{a}_{33} \Gamma_{33}, \\
& \hat{A}_{8}=\frac{2 i \delta \mu B_{q}}{\bar{E}_{q}^{4}-4 B_{q}^{2}-\delta \mu^{2} \bar{\xi}_{q}^{2}} \hat{\tau}_{2} \otimes \hat{\eta}_{3} \equiv \tilde{a}_{23} \Gamma_{23}, \\
& \hat{A}_{9}=-\frac{\delta \mu \bar{\xi}_{q} \operatorname{Re} \Delta_{0}}{\bar{E}_{q}^{4}-4 B_{q}^{2}-\delta \mu^{2} \bar{\xi}_{q}^{2}} \hat{\tau}_{3} \otimes \hat{\eta}_{1} \equiv \tilde{a}_{31} \Gamma_{31}, \\
& \hat{A}_{10}=\frac{\delta \mu \bar{\xi}_{q} \operatorname{Im} \Delta_{0}}{\bar{E}_{q}^{4}-4 B_{q}^{2}-\delta \mu^{2} \bar{\xi}_{q}^{2}} \hat{\tau}_{3} \otimes \hat{\eta}_{2} \equiv \tilde{a}_{32} \Gamma_{32}, \\
& \hat{A}_{11}=\frac{i \delta \mu \bar{\xi}_{q} \operatorname{Re} \tilde{\Delta}(\mathbf{q})}{\bar{E}_{q}^{4}-4 B_{q}^{2}-\delta \mu^{2} \bar{\xi}_{q}^{2}} \hat{\tau}_{2} \otimes \hat{\eta}_{1} \equiv \tilde{a}_{21} \Gamma_{21}, \\
& \hat{A}_{12}=-\frac{i \delta \mu \bar{\xi}_{q} \operatorname{Im} \tilde{\Delta}(\mathbf{q})}{\bar{E}_{q}^{4}-4 B_{q}^{2}-\delta \mu^{2} \bar{\xi}_{q}^{2}} \hat{\tau}_{2} \otimes \hat{\eta}_{2} \equiv \tilde{a}_{22} \Gamma_{22} .
\end{aligned}
$$

Taking these definitions into account, we write the Green's function in the block-matrix form as

$$
\hat{\mathcal{G}}_{0}\left(\omega_{n}, \mathbf{q}\right)=\left[\begin{array}{ll}
\hat{G}_{0}^{--} & \hat{G}_{0}^{-+} \\
\hat{G}_{0}^{+-} & \hat{G}_{0}^{++}
\end{array}\right] .
$$

In particular, we are interested in the correlators

$$
\begin{aligned}
\left\langle\hat{\alpha}_{-}(\mathbf{q}) \hat{\alpha}_{+}(-\mathbf{q})\right\rangle & =\left[\hat{G}_{0}^{-+}\right]_{21}=\tilde{a}_{11}-i \tilde{a}_{21}-i\left(\tilde{a}_{12}-i \tilde{a}_{22}\right) \\
& =\frac{2 B_{q} \Delta_{0}-\bar{E}_{q}^{2} \tilde{\Delta}(\mathbf{q})+\delta \mu \bar{\xi}_{q} \tilde{\Delta}(\mathbf{q})}{\bar{E}_{q}^{4}-4 B_{q}^{2}-\delta \mu^{2} \bar{\xi}_{q}^{2}}(\mathrm{C} 1
\end{aligned}
$$

and

$$
\begin{aligned}
\left\langle\hat{\alpha}_{-}(\mathbf{q}) \hat{\alpha}_{-}(-\mathbf{q})\right\rangle & =\left[\hat{G}_{0}^{--}\right]_{21}=\tilde{a}_{01}+\tilde{a}_{31}-i\left(\tilde{a}_{02}+\tilde{a}_{32}\right) \\
& =\frac{2 B_{q} \tilde{\Delta}(\mathbf{q})-\bar{E}_{q}^{2} \Delta_{0}-\delta \mu \bar{\xi}_{q} \Delta_{0}}{\bar{E}_{q}^{4}-4 B_{q}^{2}-\delta \mu^{2} \bar{\xi}_{q}^{2}} .
\end{aligned}
$$

\section{BCS gap equations}

Let us now consider the self-consistent BCS gap equations arising from the clean system previously discussed for the linear order in pairing amplitudes. Since we assume interFS and intra-FS pairings, we then obtain a pair of coupled self-consistent BCS gap equations:

$$
\begin{aligned}
\Delta_{0}= & -T \sum_{\mathbf{q}^{\prime}, \omega_{n}} V_{\text {intra }}\left(\mathbf{q}, \mathbf{q}^{\prime}\right)\left\langle\hat{\alpha}_{-}\left(\mathbf{q}^{\prime}\right) \hat{\alpha}_{-}\left(-\mathbf{q}^{\prime}\right)\right\rangle \\
= & \sum_{\mathbf{q}^{\prime}} V_{\text {intra }}\left(\mathbf{q}^{\prime}\right)\left\{\left(\frac{\Delta_{0}}{2}-\frac{2 B_{q^{\prime}} \tilde{\Delta}\left(\mathbf{q}^{\prime}\right)-\delta \mu \bar{\xi}_{q^{\prime}} \Delta_{0}}{\sqrt{4 B_{q^{\prime}}^{2}+\delta \mu^{2} \bar{\xi}_{q^{\prime}}^{2}}}\right) \mathcal{T}_{\beta}\left(\gamma_{\mathbf{q}^{\prime}}\right)\right. \\
& \left.+\left(\frac{\Delta_{0}}{2}+\frac{2 B_{q^{\prime}} \tilde{\Delta}\left(\mathbf{q}^{\prime}\right)-\delta \mu \bar{\xi}_{q^{\prime}} \Delta_{0}}{\sqrt{4 B_{q^{\prime}}^{2}+\delta \mu^{2} \bar{\xi}_{q^{\prime}}^{2}}}\right) \mathcal{T}_{\beta}\left(\Gamma_{\mathbf{q}^{\prime}}\right)\right\}, \quad(\mathrm{C} 12) \\
\tilde{\Delta}(\mathbf{q})= & -T \sum_{\mathbf{q}^{\prime}, \omega_{n}} V_{\text {inter }}\left(\mathbf{q}, \mathbf{q}^{\prime}\right)\left\langle\hat{\alpha}_{-}\left(\mathbf{q}^{\prime}\right) \hat{\alpha}_{+}\left(-\mathbf{q}^{\prime}\right)\right\rangle \\
= & \sum_{\mathbf{q}^{\prime}} V_{\text {inter }}\left(\mathbf{q}, \mathbf{q}^{\prime}\right)\left\{\left(\frac{\tilde{\Delta}\left(\mathbf{q}^{\prime}\right)}{2}-\frac{2 B_{q^{\prime}} \Delta_{0}+\delta \mu \bar{\xi}_{q^{\prime}} \tilde{\Delta}\left(\mathbf{q}^{\prime}\right)}{\sqrt{4 B_{q^{\prime}}^{2}+\delta \mu^{2} \bar{\xi}_{q^{\prime}}^{2}}}\right) \mathcal{T}_{\beta}\right. \\
& \times\left(\gamma_{\mathbf{q}^{\prime}}\right)+\left(\frac{\tilde{\Delta}\left(\mathbf{q}^{\prime}\right)}{2}+\frac{2 B_{q^{\prime}} \Delta_{0}+\delta \mu \bar{\xi}_{q^{\prime}} \tilde{\Delta}\left(\mathbf{q}^{\prime}\right)}{\sqrt{4 B_{q^{\prime}}^{2}+\delta \mu^{2} \bar{\xi}_{q^{\prime}}^{2}}}\right) \\
& \left.\times \mathcal{T}_{\beta}\left(\Gamma_{\mathbf{q}^{\prime}}\right)\right\} .
\end{aligned}
$$

Here, $\mathcal{T}_{\beta}(x) \equiv \tanh (\beta x / 2) / x$. To arrive at these expressions, we performed a partial fraction decomposition by factoring the denominator in the correlators as follows:

$$
\begin{aligned}
\bar{E}_{q}^{4} & -4 B_{q}^{2}-\delta \mu^{2} \bar{\xi}_{q}^{2} \\
& =\left(\bar{E}_{q}^{2}+\sqrt{4 B_{q}^{2}+\delta \mu^{2} \bar{\xi}_{q}^{2}}\right)\left(\bar{E}_{q}^{2}-\sqrt{4 B_{q}^{2}+\delta \mu^{2} \bar{\xi}_{q}^{2}}\right) \\
& =\left(\omega_{n}^{2}+\Gamma_{q}^{2}\right)\left(\omega_{n}^{2}+\gamma_{q}^{2}\right),
\end{aligned}
$$

where we defined the parameters

$$
\begin{aligned}
& \Gamma_{q}^{2}=\bar{\xi}_{q}^{2}+\left|\Delta_{0}\right|^{2}+|\tilde{\Delta}(\mathbf{q})|^{2}+\frac{\delta \mu^{2}}{4}+\sqrt{4 B_{q}^{2}+\delta \mu^{2} \xi_{q}^{2}}, \\
& \gamma_{q}^{2}=\bar{\xi}_{q}^{2}+\left|\Delta_{0}\right|^{2}+|\tilde{\Delta}(\mathbf{q})|^{2}+\frac{\delta \mu^{2}}{4}-\sqrt{4 B_{q}^{2}+\delta \mu^{2} \xi_{q}^{2}} .
\end{aligned}
$$

The Matsubara sums were performed by partial fraction decomposition, followed by the application of the basic identity (for $c \in \mathbb{R}$ )

$$
T \sum_{\omega_{n}} \frac{1}{\omega_{n}^{2}+c^{2}}=\frac{\tanh \left(\frac{c}{2 T}\right)}{c} .
$$

Let us now calculate the critical temperature $T_{c}=\beta_{c}^{-1}$ by imposing the condition $\Delta_{0} \rightarrow 0, \tilde{\Delta}(\mathbf{q}) \rightarrow 0$ in Eqs. (C12) and (C13). In this limit, we have $B_{q} \rightarrow 0$, and hence, the parameters defined above reduce to

$$
\begin{aligned}
\Gamma_{q} & \rightarrow \bar{\xi}_{q}+\frac{\delta \mu}{2}=v_{F} q-\mu^{-}, \\
\gamma_{q} & \rightarrow \bar{\xi}_{q}-\frac{\delta \mu}{2}=v_{F} q-\mu^{+} .
\end{aligned}
$$

The forms of the spherical harmonic and the monopole SC order parameters for the intra- and inter-FS pairings dictate the angular dependence of the pairing potentials for each channel,

$$
\begin{aligned}
V_{\text {intra }}\left(\mathbf{q}, \mathbf{q}^{\prime}\right) & =V_{0} Y_{l, m}\left(\theta_{q}, \phi_{q}\right) Y_{l, m}^{*}\left(\theta_{q^{\prime}}, \phi_{q^{\prime}}\right), \\
V_{\text {inter }}\left(\mathbf{q}, \mathbf{q}^{\prime}\right) & =\tilde{V}_{0} Y_{-1,1,0}\left(\theta_{q}, \phi_{q}\right) Y_{-1,1,0}^{*}\left(\theta_{q^{\prime}}, \phi_{q^{\prime}}\right) .
\end{aligned}
$$


Let us first consider the simplest case of an $s$-wave intranodal pairing, represented in Eq. (C17) by the spherical harmonic $l=m=0$, which is just a con- stant. Under this assumption, the coupled BCS equations, at the critical temperature $T_{c}$, reduce to the simpler expressions

$$
\begin{aligned}
& V_{0}^{-1}=\int \frac{d^{3} q}{(2 \pi)^{3}}\left[\frac{\left[1+\operatorname{sgn}\left(\delta \mu \bar{\xi}_{q}\right)\right]}{2} \frac{\tanh \left[\beta_{c}\left(\bar{\xi}_{q}-\delta \mu / 2\right)\right]}{\bar{\xi}_{q}-\delta \mu / 2}+\frac{\left[1-\operatorname{sgn}\left(\delta \mu \bar{\xi}_{q}\right)\right]}{2} \frac{\tanh \left[\beta_{c}\left(\bar{\xi}_{q}+\delta \mu / 2\right)\right]}{\bar{\xi}_{q}+\delta \mu / 2}\right], \\
& \kappa^{-1}=\int \frac{d^{3} q}{(2 \pi)^{3}}\left[\frac{\left[1-\operatorname{sgn}\left(\delta \mu \bar{\xi}_{q}\right)\right]}{2} \frac{\tanh \left[\beta_{c}\left(\bar{\xi}_{q}-\delta \mu / 2\right)\right]}{\bar{\xi}_{q}-\delta \mu / 2}+\frac{\left[1+\operatorname{sgn}\left(\delta \mu \bar{\xi}_{q}\right)\right]}{2} \frac{\tanh \left[\beta_{c}\left(\bar{\xi}_{q}+\delta \mu / 2\right)\right]}{\bar{\xi}_{q}+\delta \mu / 2}\right] .
\end{aligned}
$$

Here, we define the coefficient

$$
\kappa=\frac{\tilde{V}_{0}}{4 \pi} \int d \Omega_{q}\left|Y_{-1,1,0}\left(\theta_{q}, \phi_{q}\right)\right|^{2} .
$$

It is convenient to change integration variables in the momentum integrals defined above by introducing the density of states

$$
\rho(\xi)=\int \frac{d^{3} q}{(2 \pi)^{3}} \delta\left(\xi+\mu-v_{F} q\right)=\int \frac{d \Omega_{q}}{(2 \pi)^{3}} \int_{0}^{\infty} d q q^{2} \delta\left(\xi+\mu-v_{F} q\right)=\frac{4 \pi}{(2 \pi)^{3}} \frac{(\xi+\mu)^{2}}{\left(v_{F}\right)^{3}}
$$

to express them as energy integrals, within a symmetric interval $-\omega_{D} \leqslant \xi \leqslant \omega_{D}$, centered on the chemical potential and bounded by the physical cutoff provided by the phonon Debye frequency $\omega_{D}$. With these considerations for $\mathcal{F}\left(\Omega_{q}, \xi_{q}\right)$, which is an arbitrary function of $\mathbf{q}$, and $d \Omega_{q}=d \theta d \phi \sin \theta \cos \phi$, which is the differential solid angle, we adopt the prescription

$$
\int \frac{d^{3} q}{(2 \pi)^{3}} \mathcal{F}\left(\Omega_{q}, \xi_{q}\right)=\int \frac{d \Omega_{q}}{4 \pi} \int_{-\omega_{D}}^{\omega_{D}} d \xi \rho(\xi) \mathcal{F}\left(\Omega_{q}, \xi\right) \sim \rho(\mu) \int \frac{d \Omega_{q}}{4 \pi} \int_{-\omega_{D}}^{\omega_{D}} d \xi \mathcal{F}\left(\Omega_{q}, \xi\right)
$$

By converting the momentum integral into the one over energy through the density of states, as described above, Eqs. (C18) and (C19) reduce to

$$
\begin{aligned}
\lambda_{\text {intra }}^{-1}= & \int_{-\omega_{D}}^{\omega_{D}} d \xi\left[\frac{[1+\operatorname{sgn}(\delta \mu \xi)]}{2} \frac{\tanh \left[\beta_{c}(\xi-\delta \mu / 2)\right]}{\xi-\delta \mu / 2}\right. \\
& \left.+\frac{[1-\operatorname{sgn}(\delta \mu \xi)]}{2} \frac{\tanh \left[\beta_{c}(\xi+\delta \mu / 2)\right]}{\xi+\delta \mu / 2}\right], \quad(\mathrm{C} 23) \\
\lambda_{\text {inter }}^{-1}= & \int_{-\omega_{D}}^{\omega_{D}} d \xi\left[\frac{[1-\operatorname{sgn}(\delta \mu \xi)]}{2} \frac{\tanh \left[\beta_{c}(\xi-\delta \mu / 2)\right]}{\xi-\delta \mu / 2}\right. \\
& \left.+\frac{[1+\operatorname{sgn}(\delta \mu \xi)]}{2} \frac{\tanh \left[\beta_{c}(\xi+\delta \mu / 2)\right]}{\xi+\delta \mu / 2}\right] .
\end{aligned}
$$

Here, we define the effective couplings

$$
\begin{aligned}
\lambda_{\text {inter }} & =V_{0} \rho(\mu), \\
\lambda_{\text {intra }} & =\kappa \rho(\mu),
\end{aligned}
$$

with the density of states $\rho(\mu)$, as defined in Eq. (C21), evaluated at the average chemical potential.

Let us now consider the $T=0$ BCS gap equation for a more general intra-FS pairing as defined in Eq. (C17). For convenience, we introduce the notation

$$
\begin{aligned}
& \Delta_{\text {inter }}(\mathbf{q})=\bar{\Delta}_{0} d_{\text {inter }}(\theta) e^{i(m+q) \phi}, \\
& \Delta_{\text {intra }}(\mathbf{q})=\Delta_{l m, 0} d_{\text {intra }}(\theta) e^{i m^{\prime} \phi},
\end{aligned}
$$

where for the spherical harmonics we write $Y_{l, m^{\prime}}(\theta, \phi)=$ $e^{i m^{\prime} \phi} f_{l}(\theta)$ and choose $m^{\prime}>0$ without loss of generality. The form of the monopole harmonic is given by Eq. (20), which we rewrite as $Y_{q, j, m}=e^{i(m+q) \phi} g(\theta)$, with other indices omitted for clarity, and we set $m+q>0$ since the choice $m+q<0$ is gauge equivalent, as shown below.

The generalization of Eqs. (C12) and (C13), obtained from the Green's function in Eq. (C6), retaining also nonlinear terms in the pairing amplitudes, then reads

$$
\begin{aligned}
\Delta_{\text {intra }}(\mathbf{q})= & -T \sum_{\mathbf{q}^{\prime}, \omega_{n}} V_{\text {intra }}\left(\mathbf{q}, \mathbf{q}^{\prime}\right)\left\langle\hat{\alpha}_{-}\left(\mathbf{q}^{\prime}\right) \hat{\alpha}_{-}\left(-\mathbf{q}^{\prime}\right)\right\rangle \\
= & \sum_{\mathbf{q}^{\prime}} V_{\text {intra }}\left(\mathbf{q}, \mathbf{q}^{\prime}\right)\left\{\left(\frac{\Delta_{\text {intra }}\left(\mathbf{q}^{\prime}\right)}{2}-\frac{2 B_{q^{\prime}} \Delta_{\text {inter }}\left(\mathbf{q}^{\prime}\right)-\delta \mu \bar{\xi}_{q^{\prime}} \Delta_{\text {intra }}\left(\mathbf{q}^{\prime}\right)}{\sqrt{4 B_{q^{\prime}}^{2}+\delta \mu^{2} \bar{\xi}_{q^{\prime}}^{2}}}\right) \frac{\tanh \left(\beta \gamma_{\mathbf{q}^{\prime}} / 2\right)}{\gamma_{\mathbf{q}^{\prime}}}\right. \\
& \left.+\left(\frac{\Delta_{\text {intra }}\left(\mathbf{q}^{\prime}\right)}{2}+\frac{2 B_{q^{\prime}} \Delta_{\text {inter }}\left(\mathbf{q}^{\prime}\right)-\delta \mu \bar{\xi}_{q^{\prime}} \Delta_{\text {intra }}\left(\mathbf{q}^{\prime}\right)}{\sqrt{4 B_{q^{\prime}}^{2}+\delta \mu^{2} \bar{\xi}_{q^{\prime}}^{2}}}\right) \frac{\tanh \left(\beta \Gamma_{\mathbf{q}^{\prime}} / 2\right)}{\Gamma_{\mathbf{q}^{\prime}}}\right\} \\
\Delta_{\text {inter }}(\mathbf{q})= & -T \sum_{\mathbf{q}^{\prime}, \omega_{n}} V_{\text {inter }}\left(\mathbf{q}, \mathbf{q}^{\prime}\right)\left\langle\hat{\alpha}_{-}\left(\mathbf{q}^{\prime}\right) \hat{\alpha}_{+}\left(-\mathbf{q}^{\prime}\right)\right\rangle
\end{aligned}
$$




$$
\begin{aligned}
= & \sum_{\mathbf{q}^{\prime}} V_{\text {inter }}\left(\mathbf{q}, \mathbf{q}^{\prime}\right)\left\{\left(\frac{\Delta_{\text {inter }}\left(\mathbf{q}^{\prime}\right)}{2}-\frac{2 B_{q^{\prime}} \Delta_{\text {intra }}\left(\mathbf{q}^{\prime}\right)+\delta \mu \bar{\xi}_{q^{\prime}} \Delta_{\text {inter }}\left(\mathbf{q}^{\prime}\right)}{\sqrt{4 B_{q^{\prime}}^{2}+\delta \mu^{2} \bar{\xi}_{q^{\prime}}^{2}}}\right) \frac{\tanh \left(\beta \gamma_{\mathbf{q}^{\prime}} / 2\right)}{\gamma_{\mathbf{q}^{\prime}}}\right. \\
& \left.+\left(\frac{\Delta_{\text {inter }}\left(\mathbf{q}^{\prime}\right)}{2}+\frac{2 B_{q^{\prime}} \Delta_{\text {intra }}\left(\mathbf{q}^{\prime}\right)+\delta \mu \bar{\xi}_{q^{\prime}} \Delta_{\text {inter }}\left(\mathbf{q}^{\prime}\right)}{\sqrt{4 B_{q^{\prime}}^{2}+\delta \mu^{2} \bar{\xi}_{q^{\prime}}^{2}}}\right) \frac{\tanh \left(\beta \Gamma_{\mathbf{q}^{\prime}} / 2\right)}{\Gamma_{\mathbf{q}^{\prime}}}\right\}
\end{aligned}
$$

with the parameter $B_{q}$ now written in the form

$$
B_{q}=\operatorname{Re} \Delta_{\text {inter }}(\mathbf{q}) \operatorname{Re} \Delta_{\text {intra }}(\mathbf{q})+\operatorname{Im} \Delta_{\text {inter }}(\mathbf{q}) \operatorname{Im} \Delta_{\text {intra }}(\mathbf{q}) .
$$

As in the first example, we are interested in the vicinity of the phase boundary, where $\left|\Delta_{\text {inter }}\right| \ll 1$ and $\left|\Delta_{\text {intra }}\right| \ll 1$, such that we retain only linear terms in the numerators of Eqs. (C27) and (C28).

After integrating over $|\xi| \leqslant \omega_{D}$ in Eqs. (C12) and (C13), using the identity in Eq. (C22) and the result

$$
\begin{aligned}
& \int_{-\omega_{D}}^{\omega_{D}} d \xi \frac{\rho(\xi)}{\sqrt{\xi^{2}+\left|\Delta_{\text {inter }}\right|^{2}+\left|\Delta_{\text {intra }}\right|^{2} \pm 2\left[\operatorname{Re} \Delta_{\text {inter }} \operatorname{Re} \Delta_{\text {intra }}+\operatorname{Im} \Delta_{\text {inter }} \operatorname{Im} \Delta_{\text {intra }}\right.}} \\
& \quad=2 \rho(\mu)\left\{\ln \left(2 \omega_{D}\right)-\frac{1}{2} \ln \left[\left|\Delta_{\text {inter }}\right|^{2}+\left|\Delta_{\text {intra }}\right|^{2} \pm 2\left(\operatorname{Re} \Delta_{\text {inter }} \operatorname{Re} \Delta_{\text {intra }}+\operatorname{Im} \Delta_{\text {inter }} \operatorname{Im} \Delta_{\text {intra }}\right)\right]\right\}
\end{aligned}
$$

we obtain the corresponding generalized zero-temperature BCS gap equations in Eq. (12) in the main text.

\section{APPENDIX D: PHASE BOUNDARY BETWEEN THE $S$-WAVE AND THE HYBRID SUPERCONDUCTOR}

To further corroborate the possibility of phase coexistence close to the line given by Eq. (27) [see also Fig. 1(a)], defined by $\theta_{0}=\pi / 2\left(\Delta_{s}=\bar{\Delta}_{0}\right)$, we expand the expressions in Eq. (26) up to third order with respect to the small parameter $x=\frac{\pi}{2}-\theta_{0} \ll 1$ and substitute them into Eq. (23) to obtain

$$
\begin{aligned}
\lambda_{s}^{-1} & =2 \ln \left(2 \omega_{D}\right)-\ln \left(\Delta_{s}^{2}\right)-\frac{2}{3} x^{3}, \\
\lambda_{\text {inter }}^{-1} & =\frac{4}{3} \ln \left(2 \omega_{D}\right)-\frac{2}{3} \ln \left(\Delta_{s}^{2}\right)-\frac{2}{3} x^{2}+\frac{4}{3} x^{3} .
\end{aligned}
$$

We notice that for $x \ll 1$,

$$
\lambda_{\text {inter }}^{-1}-\frac{2}{3} \lambda_{s}^{-1}=-\frac{2}{3} x^{2}+\frac{16}{9} x^{3}<0,
$$

and hence, the phase coexistence is possible. However, the positive cubic term implies that eventually, for a sufficiently strong inter-FS pairing, the monopole SC phase dominates, as explicitly found in the analysis presented around Eq. (27) in the main text.

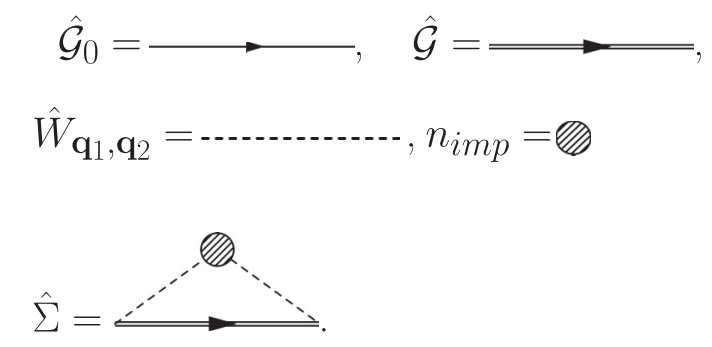

FIG. 4. Feynman diagram for the impurity-averaged self-energy in the first Born approximation. The different symbols are also displayed.

\section{APPENDIX E: IMPURITY SCATTERING}

We now address the effect of scattering by randomly distributed, nonmagnetic impurities with a concentration $n_{\mathrm{imp}}$. Within the first Born approximation, this can be captured through an averaged self-energy matrix of the form [33]

$$
\hat{\Sigma}\left(\omega_{n}, \mathbf{q}\right)=n_{\text {imp }} \sum_{\mathbf{q}_{1}} \hat{W}_{\mathbf{q}, \mathbf{q}_{1}} \hat{\mathcal{G}}\left(\omega_{n}, \mathbf{q}_{1}\right) \hat{W}_{\mathbf{q}_{1}, \mathbf{q}},
$$

where $\hat{\mathcal{G}}\left(\omega_{n}, \mathbf{q}\right)$ is the full interacting Green's function matrix, arising from the solution to the Dyson equation

$$
\hat{\mathcal{G}}^{-1}\left(\omega_{n}, \mathbf{q}\right)=\hat{\mathcal{G}}_{0}^{-1}\left(\omega_{n}, \mathbf{q}\right)-\hat{\Sigma}\left(\omega_{n}, \mathbf{q}\right) .
$$

The Feynman diagrams representing the bare and dressed Green's functions, as well as the self-energy within the first Born approximation [33], are depicted in Fig. 4. The self-consistent solution for the Dyson equation (E2) can be represented diagrammatically as shown in Fig. 5.

We assume, for simplicity, that the elements of the scattering potential matrix are independent of momenta and include both inter- and intra-FS scattering processes:

$$
\hat{W}_{\mathbf{q}, \mathbf{q}_{1}}=u \hat{\tau}_{1} \otimes \hat{\eta}_{0}+v \hat{\tau}_{0} \otimes \hat{\eta}_{3} .
$$

The Dyson equation can be solved by assuming the ansatz that the fully dressed Green's function possesses the same structure as the one in the clean case, but with renormalized parameters $\omega_{n, R}, \Delta_{R}(\mathbf{q}), \Delta_{0, R}$, and $\xi_{n, q, R}$. In the following, we consider Eq. (C9) for $\hat{\mathcal{G}}_{0}\left(\omega_{n}, \mathbf{q}\right)$, neglecting the small

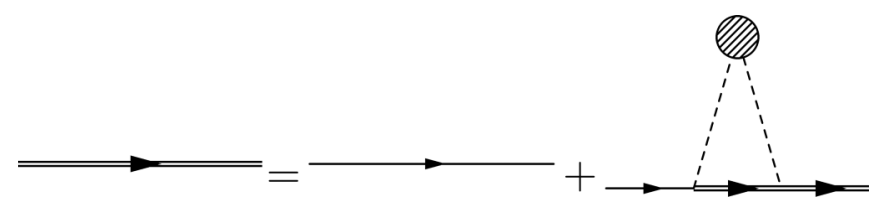

FIG. 5. The Dyson equation for the first Born approximation. The symbols are described in Fig. 4. 
nodal asymmetry in the chemical potentials $\delta \mu=0$. We repeat a matrix analysis similar to that in Appendices B and
$\mathrm{C}$ and solve the Dyson equation to obtain the renormalized parameters in the form

$$
\begin{aligned}
i \omega_{n, R} & =i \omega_{n}-n_{\text {imp }} \int \frac{d^{3} k}{(2 \pi)^{3}}\left\{-\left(u^{2}+v^{2}\right) \frac{i \omega_{n} E_{k}^{2}}{E_{k}^{4}-4 B_{k}^{2}}+2 u v \frac{B_{k} \xi_{k}}{E_{k}^{4}-4 B_{k}^{2}}\right\} \\
\xi_{n, q, R} & =\xi_{q}+n_{\text {imp }} \int \frac{d^{3} k}{(2 \pi)^{3}}\left\{-\left(u^{2}+v^{2}\right) \frac{\xi_{k} E_{k}^{2}}{E_{k}^{4}-4 B_{k}^{2}}+2 u v \frac{B_{k} i \omega_{n}}{E_{k}^{4}-4 B_{k}^{2}}\right\} \\
\Delta_{0, R} & =\Delta_{0}+n_{\text {imp }} \int \frac{d^{3} k}{(2 \pi)^{3}}\left(u^{2}-v^{2}\right) \frac{2 B_{k} \tilde{\Delta}(\mathbf{k})-E_{k}^{2} \Delta_{0}}{E_{k}^{4}-4 B_{k}^{2}} .
\end{aligned}
$$

Let us focus first on the intra-FS scattering case by setting $u=0$. At the critical temperature $\bar{T}_{c}$, we apply the condition $\Delta_{0} \rightarrow 0$, such that in this limit the parameters $E_{k} \rightarrow \xi_{k}$ and $B_{k} \rightarrow 0$. Therefore, the linearized form for the renormalized Matsubara frequencies reduces to

$$
\begin{aligned}
\omega_{n, R} & =\omega_{n}+\omega_{n} n_{\text {imp }} v^{2} \int \frac{d^{3} k}{(2 \pi)^{3}} \frac{\xi_{k}^{2}+\omega_{n}^{2}}{\left(\xi_{k}^{2}+\omega_{n}^{2}\right)^{2}} \\
& =\omega_{n}+n_{\text {imp }} v^{2} \omega_{n} \int_{-\omega_{D}}^{\omega_{D}} d \xi \frac{\rho(\xi)}{\omega_{n}^{2}+\xi^{2}} \\
& =\omega_{n}+2 n_{\text {imp }} v^{2} \rho(\mu) \arctan \left(\omega_{D} / \omega_{n}\right) \\
& =\omega_{n}\left(1+\frac{1}{2 \tau_{\text {intra }}\left|\omega_{n}\right|}\right),
\end{aligned}
$$

where in the last step, we assumed $\omega_{D} \gg T_{c}$, such that $\arctan \left(\omega_{D} / \omega_{n}\right) \rightarrow(\pi / 2) \operatorname{sgn}\left(\omega_{n}\right)$. We have also defined the intra-FS scattering relaxation time

$$
\tau_{\text {intra }}^{-1}=2 \pi n_{\text {imp }} v^{2} \rho(\mu) .
$$

Similarly, the equation for the renormalized band dispersion reduces to

$$
\begin{aligned}
\xi_{n, q, R} & =\xi_{q}-v^{2} n_{\text {imp }} \int_{-\omega_{D}}^{\omega_{D}} d \xi \frac{\xi \rho(\xi)}{\omega_{n}^{2}+\xi^{2}} \\
& =\xi_{q}
\end{aligned}
$$

since the integrand is odd and hence the correction to the band structure vanishes. Finally, the renormalized pairing reduces to

$$
\begin{aligned}
\Delta_{0, R} & =\Delta_{0}+2 n_{\mathrm{imp}} v^{2} \Delta_{0} \rho(\mu) \int_{0}^{\omega_{D}} \frac{d \xi}{\omega_{n}^{2}+\xi^{2}} \\
& =\Delta_{0}\left(1+\frac{1}{2 \tau_{\text {intra }}\left|\omega_{n}\right|}\right) .
\end{aligned}
$$

If one considers now the effect of inter-FS scattering, i.e,. by setting $u \neq 0, v \neq 0$, the same analysis as above leads to

$$
\begin{aligned}
& \Delta_{0, R}=\Delta_{0}\left[1+\frac{1}{2}\left(\tau_{\text {intra }}^{-1}-\tau_{\text {inter }}^{-1}\right)\left|\omega_{n}\right|^{-1}\right] \\
& \omega_{n, R}=\omega_{n}\left[1+\frac{1}{2}\left(\tau_{\text {intra }}^{-1}+\tau_{\text {inter }}^{-1}\right)\left|\omega_{n}\right|^{-1}\right],
\end{aligned}
$$

where we define

$$
\tau_{\text {inter }}^{-1}=2 \pi n_{\text {imp }} u^{2} \rho(\mu) .
$$

\section{BCS gap equation}

The BCS gap equations that include the effect of scattering by random impurities are given by

$$
\begin{aligned}
\Delta_{0} & =V_{0} \sum_{n} \int \frac{d^{3} q}{(2 \pi)^{3}} \frac{\Delta_{0, R}}{\omega_{n, R}^{2}+\xi_{q, R}^{2}}=V_{0} \sum_{n} \int \frac{d^{3} q}{(2 \pi)^{3}} \frac{\Delta_{0}\left[1+\frac{1}{2}\left(\tau_{\text {intra }}^{-1}-\tau_{\text {inter }}^{-1}\right)\left|\omega_{n}\right|^{-1}\right]}{\omega_{n}^{2}\left[1+\frac{1}{2}\left(\tau_{\text {intra }}^{-1}+\tau_{\text {inter }}^{-1}\right)\left|\omega_{n}\right|^{-1}\right]^{2}+\xi_{q}^{2}} \\
& =2 V_{0} \rho(\mu) \sum_{n} \int_{0}^{\omega_{D}} d \xi \frac{\Delta_{0}\left[1+\frac{1}{2}\left(\tau_{\text {intra }}^{-1}-\tau_{\text {inter }}^{-1}\right)\left|\omega_{n}\right|^{-1}\right]}{\omega_{n}^{2}\left[1+\frac{1}{2}\left(\tau_{\text {intra }}^{-1}+\tau_{\text {inter }}^{-1}\right)\left|\omega_{n}\right|^{-1}\right]^{2}+\xi^{2}} \\
& =2 V_{0} \rho(\mu) \sum_{n} \frac{\Delta_{0}\left[1+\frac{1}{2}\left(\tau_{\text {intra }}^{-1}-\tau_{\text {inter }}^{-1}\right)\left|\omega_{n}\right|^{-1}\right]}{\left|\omega_{n}\right|\left[1+\frac{1}{2}\left(\tau_{\text {intra }}^{-1}+\tau_{\text {inter }}^{-1}\right)\left|\omega_{n}\right|^{-1}\right]} \arctan \left(\frac{\omega_{D}}{\left|\omega_{n}\right|\left[1+\frac{1}{2}\left(\tau_{\text {intra }}^{-1}+\tau_{\text {inter }}^{-1}\right)\left|\omega_{n}\right|^{-1}\right]}\right) .
\end{aligned}
$$

Assuming, as before, that $\omega_{D} \gg T_{c}$, the previous expression simplifies to

$$
1=V_{0} \rho(\mu) \pi \sum_{n} \frac{1}{\left|\omega_{n}\right|+\tau_{\text {inter }}^{-1}}=\frac{V_{0} \rho(\mu)}{T_{c}}\left[\ln \left(\frac{\Gamma_{c}}{2 \pi T_{c}}\right)-\psi\left(\frac{1}{2}+\frac{1}{2 \pi \tau_{\text {inter }} T_{c}}\right)\right],
$$

where $\Gamma_{c}$ is an upper cutoff for the Matsubara frequency sum and $\psi(x)$ is the digamma function. 
A similar analysis can now be performed for the monopole SC pairing. In this case, the vortex part protects the gap function from renormalization,

$$
\tilde{\Delta}_{R}(\mathbf{q})=\tilde{\Delta}(\mathbf{q}) .
$$

Therefore, the corresponding BCS gap equation for the monopole $\mathrm{SC}$ pairing becomes

$$
\begin{gathered}
\tilde{\Delta}_{0}=\kappa \sum_{n} \int \frac{d^{3} q}{(2 \pi)^{3}} \frac{\tilde{\Delta}_{0}}{\bar{\omega}_{n}^{2}+\xi_{q}^{2}} \\
=2 \kappa \rho(\mu) \sum_{n} \int_{\xi} \frac{\tilde{\Delta}_{0}}{\omega_{n}^{2}\left[1+\frac{1}{2}\left(\tau_{\text {inter }}^{-1}+\tau_{\text {intra }}^{-1}\right)\left|\omega_{n}\right|^{-1}\right]^{2}+\xi^{2}},
\end{gathered}
$$

where $\int_{\xi} \equiv \int_{0}^{\omega_{D}} d \xi$ and $\kappa$ is defined as in Eq. (C20). Analogous to the previous case, i.e., $\omega_{D} \gg T_{c}$, the integral and Matsubara sum can be performed to yield the corresponding equation for the critical temperature of the monopole pairing:

$$
\frac{T_{c}}{\kappa \rho(\mu)}=\ln \left(\frac{\Gamma_{c}}{2 \pi T_{c}}\right)-\psi\left(\frac{1}{2}+\frac{\tau_{\text {intra }}^{-1}+\tau_{\text {inter }}^{-1}}{4 \pi T_{c}}\right) .
$$

Figure 3 in the main text is obtained by using Eqs. (E12) and (E16) and shows the dependence of the phase boundary on the intra-FS inverse scattering time for various choices of $\tau_{\text {inter. }}^{-1}$. At the phase boundary the critical temperatures for the two pairings are equal.
[1] G. E. Volovik, The Universe in a Helium Droplet (Oxford University Press, New York, 2003).

[2] T. O. Wehling, A. M. Black-Schaffer, and A. V. Balatsky, Adv. Phys. 63, 1 (2014).

[3] B. J. Yang and N. Nagaosa, Nat. Commun. 5, 4898 (2014).

[4] C.-K. Chiu, J. C. Y. Teo, A. P. Schnyder, and S. Ryu, Rev. Mod. Phys. 88, 035005 (2016).

[5] B. Bradlyn, J. Cano, Z. Wang, M. G. Vergniory, C. Felser, R. J. Cava, and B. A. Bernevig, Science 353, aaf5037 (2016).

[6] B. J. Wieder and C. L. Kane, Phys. Rev. B 94, 155108 (2016).

[7] A. A. Burkov, Nat. Mater. 15, 1145 (2016).

[8] S. Jia, S.-Y. Xu, and M. Z. Hasan, Nat. Mater. 15, 1140 (2016).

[9] M. Z. Hasan, S.-Y. Xu, I. Beloposky, and S.-M. Huang, Annu. Rev. Condens. Matter Phys. 8, 289 (2017).

[10] N. P. Armitage, E. J. Mele, and A. Vishwanath, Rev. Mod. Phys. 90, 015001 (2018).

[11] B. A. Bernevig, H. Weng, Z. Fang, and X. Dai, J. Phys. Soc. Jpn. 87, 041001 (2018).

[12] S.-Y. Xu, I. Belopolski, N. Alidoust, M. Neupane, G. Bian, C. Zhang, R. Sankar, G. Chang, Z. Yuan, C.-C. Lee, S.-M. Huang, H. Zheng, J. Ma, D. S. Sanchez, B. K. Wang, A. Bansil, F. Chou, P. P. Shibayev, H. Lin, S. Jia, and M. Z. Hasan, Science 349, 613 (2015).

[13] B. Q. Lv, H. M. Weng, B. B. Fu, X. P. Wang, H. Miao, J. Ma, P. Richard, X. C. Huang, L. X. Zhao, G. F. Chen, Z. Fang, X. Dai, T. Qian, and H. Ding, Phys. Rev. X 5, 031013 (2015).

[14] C.-L. Zhang, Z. Yuan, Q.-D. Jiang, B. Tong, C. Zhang, X. C. Xie, and S. Jia, Phys. Rev. B 95, 085202 (2017).

[15] S.-Y. Xu, N. Alidoust, I. Belopolski, Z. Yuan, G. Bian, T.-R. Chang, H. Zheng, V. N. Strocov, D. S. Sanchez, G. Chang, C. Zhang, D. Mou, Y. Wu, L. Huang, C.-C. Lee, S.-M. Huang, B. K. Wang, A. Bansil, H.-T. Jeng, T. Neupert et al., Nat. Phys. 11, 748 (2015).

[16] Z. Wang and S.-C. Zhang, Phys. Rev. B 87, 161107(R) (2013).

[17] B. Roy and J. D. Sau, Phys. Rev. B 92, 125141 (2015).

[18] Y. You, G. Y. Cho, and T. L. Hughes, Phys. Rev. B 94, 085102 (2016).
[19] B. Roy, P. Goswami, and V. Juričić, Phys. Rev. B 95, 201102(R) (2017).

[20] J. Gooth, B. Bradlyn, S. Honnali, C. Schindler, N. Kumar, J. Noky, Y. Qi, C. Shekhar, Y. Sun, Z. Wang, B. A. Bernevig, and C. Felser, Nature (London) 575, 315 (2019).

[21] G. E. Volovik, JETP Lett. 46, 98 (1987).

[22] S. Murakami and N. Nagaosa, Phys. Rev. Lett. 90, 057002 (2003).

[23] T. Meng and L. Balents, Phys. Rev. B 86, 054504 (2012).

[24] G.-Y. Cho, J. H. Bardarson, Y.-M. Lu, and J. E. Moore, Phys. Rev. B 86, 214514 (2012).

[25] S. A. Yang, H. Pan, and F. Zhang, Phys. Rev. Lett. 113, 046401 (2014)

[26] A. P. Schnyder and P. M. R. Brydon, J. Phys.: Condens. Matter 27, 243201 (2015).

[27] G. Bednik, A. A. Zyuzin, and A. A. Burkov, Phys. Rev. B 92, 035153 (2015).

[28] Y. Li and F. D. M. Haldane, Phys. Rev. Lett. 120, 067003 (2018).

[29] C. Sun, S.-P. Lee, and Y. Li, arXiv:1909.04179.

[30] T. T. Wu and C. N. Yang, Nucl. Phys. B 107, 365 (1976).

[31] F. D. M. Haldane, Phys. Rev. Lett. 51, 605 (1983).

[32] K. A. Musaelian, J. Betouras, A. V. Chubukov, and R. Joynt, Phys. Rev. B 53, 3598 (1996).

[33] J. Rammer and H. Smith, Rev. Mod. Phys. 58, 323 (1986).

[34] P. W. Anderson, J. Phys. Chem. Solid 11, 26 (1959).

[35] A. Ramires, D. F. Agterberg, and M. Sigrist, Phys. Rev. B 98, 024501 (2018).

[36] L. Andersen, A. Ramires, Z. Wang, T. Lorenz, and Y. Ando, Sci. Adv. 6, eaay6502 (2020).

[37] I. M. Hayes, D. S. Wei, T. Metz, J. Zhang, Y. S. Eo, S. Ran, S. R Saha, J. Collini, N. P. Butch, D. F. Agterberg, A. Kapitulnik, and J. Paglione, arXiv:2002.02539.

[38] E. Bobrow, C. Sun, and Y. Li, Phys. Rev. Research 2, 012078(R) (2020).

[39] A. V. Balatsky, I. Vekhter, and J.-X. Zhu, Rev. Mod. Phys. 78, 373 (2006). 